\title{
Structural Organization of the Corpus Callosum Predicts Attentional Shifts after Continuous Theta Burst Stimulation
}

\author{
@i-Magdalena Chechlacz, ${ }^{1}$ Glyn W. Humphreys, ${ }^{1}$ Stamatios N. Sotiropoulos, ${ }^{2}$ Christopher Kennard, ${ }^{3}$ and Dario Cazzoli3 ${ }^{3,4}$ \\ ${ }^{1}$ Department of Experimental Psychology, University of Oxford, Oxford OX1 3UD, United Kingdom, ${ }^{2}$ FMRIB Centre and ${ }^{3}$ Nuffield Department of Clinical \\ Neurosciences, University of Oxford, Oxford OX3 9UD, United Kingdom, and ${ }^{4}$ Gerontechnology and Rehabilitation Group, University of Bern, 3010 Bern, \\ Switzerland
}

Repetitive transcranial magnetic stimulation (rTMS) applied over the right posterior parietal cortex (PPC) in healthy participants has been shown to trigger a significant rightward shift in the spatial allocation of visual attention, temporarily mimicking spatial deficits observed in neglect. In contrast, rTMS applied over the left PPC triggers a weaker or null attentional shift. However, large interindividual differences in responses to rTMS have been reported. Studies measuring changes in brain activation suggest that the effects of rTMS may depend on both interhemispheric and intrahemispheric interactions between cortical loci controlling visual attention. Here, we investigated whether variability in the structural organization of human white matter pathways subserving visual attention, as assessed by diffusion magnetic resonance imaging and tractography, could explain interindividual differences in the effects of rTMS. Most participants showed a rightward shift in the allocation of spatial attention after rTMS over the right intraparietal sulcus (IPS), but the size of this effect varied largely across participants. Conversely, rTMS over the left IPS resulted in strikingly opposed individual responses, with some participants responding with rightward and some with leftward attentional shifts. We demonstrate that microstructural and macrostructural variability within the corpus callosum, consistent with differential effects on cross-hemispheric interactions, predicts both the extent and the direction of the response to rTMS. Together, our findings suggest that the corpus callosum may have a dual inhibitory and excitatory function in maintaining the interhemispheric dynamics that underlie the allocation of spatial attention.

Key words: corpus callosum; diffusion tractography; individual differences; neglect; spatial attention; TMS

Significance Statement

The posterior parietal cortex (PPC) controls allocation of attention across left versus right visual fields. Damage to this area results in neglect, characterized by a lack of spatial awareness of the side of space contralateral to the brain injury. Transcranial magnetic stimulation over the PPC is used to study cognitive mechanisms of spatial attention and to examine the potential of this technique to treat neglect. However, large individual differences in behavioral responses to stimulation have been reported. We demonstrate that the variability in the structural organization of the corpus callosum accounts for these differences. Our findings suggest novel dual mechanism of the corpus callosum function in spatial attention and have broader implications for the use of stimulation in neglect rehabilitation.

\section{Introduction}

Visual attention is composed of multiple components, including those that mediate spatial selection. There is a considerable clin-

Received July 9, 2015; revised 0ct. 7, 2015; accepted Oct. 14, 2015.

Author contributions: M.C. and D.C. designed research; M.C. and D.C. performed research; G.W.H., S.N.S., and C.K. contributed unpublished reagents/analytic tools; M.C. and D.C. analyzed data; M.C., G.W.H., and D.C. wrote the paper.

This work was supported by funding from the National Institute of Health Research Oxford Cognitive Health Clinical Research Facility (G.W.H.) and Oxford Biomedical Research Centre based at the Oxford University Hospitals National Health Service Trust and the University of Oxford (C.K.). M.C. holds a British Academy Postdoctoral Fellowship. D.C. holds a Swiss National Science Foundation Ambizione Fellowship.

The authors declare no competing financial interests.

Correspondence should be addressed to Magdalena Chechlacz, Department of Experimental Psychology, Oxford University, 9 South Parks Road, 0xford 0X1 3UD, UK. E-mail: magdalena.chechlacz@psy.ox.ac.uk. ical interest in spatial attention due to striking deficits such as unilateral neglect that are typically found after damage to the right hemisphere, which results in an abnormal attentional bias to the right. In contrast to this, healthy participants show a much smaller attentional bias to the left, so-called pseudoneglect (Heilman and Valenstein, 1979; Bowers and Heilman, 1980; Driver and Mattingley, 1998; Vallar, 1998; McCourt and Jewell, 1999; Halligan et al., 2003; Sosa et al., 2010). The most commonly accepted neural account of these spatial biases proposes that there is an asymmetrical organization of visual attentional networks in

DOI:10.1523/JNEUROSCI.2610-15.2015

Copyright $\odot 2015$ the authors $\quad 0270-6474 / 15 / 3515353-16 \$ 15.00 / 0$ 
the brain, with a right hemispheric dominance (for review, see Corbetta and Shulman, 2011). To explain this asymmetry, a hemispheric rivalry model holds that, whereas each hemisphere controls spatial attention within the contralateral hemifield, the contralateral bias of the right hemisphere is stronger than the one of the left (Kinsbourne, 1977, 1987, 1993). This model speculates that the hemispheres inhibit each other and compete for directing attention toward the contralateral part of space. Therefore, the preferential activation within the dominant right hemisphere results in pseudoneglect in healthy individuals, whereas the right hemisphere damage creates an interhemispheric imbalance in attention because the intact left hemisphere is released from inhibition and becomes hyperactive, resulting in neglect (Kinsbourne, 1977, 1987; Corbetta et al., 2005). Although Kinsbourne's model relies purely on inhibitory functions operating across the corpus callosum, there is also a substantial body of evidence suggesting excitatory connections across this structure (for review, see Bloom and Hynd, 2005).

Repetitive transcranial magnetic stimulation (rTMS) studies support the notion of mutual hemispheric inhibition and right hemisphere dominance in attention. rTMS applied over the right posterior parietal cortex (PPC) in healthy individual triggers significant shifts in the allocation of visual attention, temporarily mimicking the spatial deficits observed in neglect (Fierro et al., 2000; Hilgetag et al., 2001; Hung et al., 2005; Sack et al., 2007; Cazzoli et al., 2009; for review, see Szczepanski and Kastner, 2009). In contrast, rTMS applied over the left PPC triggers either weak or null attentional shifts in healthy participants (Fierro et al., 2000; Hilgetag et al., 2001; Bjoertomt et al., 2002; Hung et al., 2005; Dambeck et al., 2006; Sack et al., 2007; Cazzoli et al., 2009; but see Battelli et al., 2009; Szczepanski and Kastner, 2013). Nevertheless, rTMS applied over the left hemisphere has been used successfully to ameliorate neglect symptoms, presumably by reducing the hyperexcitability of the intact left hemisphere (for review, see Cazzoli et al., 2010). Although the above results have been found at a group level, large, individual differences in responses to stimulation have been reported in both healthy participants and patients (Szczepanski and Kastner, 2013; Rizk et al., 2013; Plow et al., 2014; see also review by Nicolo et al., 2015). An understanding of how individual differences in behavioral responses to stimulation come about is of high importance, particularly if rTMS is to be used as clinical intervention to treat neglect (Cazzoli et al., 2012; Koch et al., 2012).

In the current study, we investigated whether structural variability in the organization of white matter (WM) pathways could explain individual differences in the effect of rTMS applied over the right and the left posterior intraparietal sulcus (IPS) on the spatial allocation of visual attention. We chose to stimulate the posterior IPS, a cortical region within the dorsal attention network with robust interhemispheric and intrahemispheric connections (Mesulam, 1990; Corbetta and Shulman, 2002; Shulman et al., 2010; Szczepanski et al., 2010; Corbetta and Shulman, 2011; Gillebert et al., 2011; Thiebaut de Schotten et al., 2011a). Based on previous research, we predicted that the differential effects of rTMS could be a consequence of variance either in the commissural pathways interconnecting the left and right hemispheres and/or in long association frontoparietal pathways within each hemisphere. To explore these predictions, we performed analyses of microstructural and macrostructural metrics of WM using whole-brain tract-based spatial statistics combined with more focused analyses based on tractography-estimated WM bundles.
Table 1. Participants' demographic data

\begin{tabular}{|c|c|c|c|}
\hline Participant & Age (y) & Sex & Handedness $^{a}$ \\
\hline P01 & 24 & $\mathrm{~F}$ & 85 \\
\hline P02 & 28 & $\mathrm{~F}$ & 73 \\
\hline P03 & 33 & $\mathrm{~F}$ & -71 \\
\hline P04 & 20 & M & 87 \\
\hline P05 & 21 & $\mathrm{~F}$ & 100 \\
\hline P06 & 24 & $\mathrm{~F}$ & 82 \\
\hline P07 & 36 & M & 100 \\
\hline P08 & 26 & M & 53 \\
\hline P09 & 36 & $\mathrm{~F}$ & -67 \\
\hline P10 & 28 & $\mathrm{~F}$ & 39 \\
\hline P11 & 20 & M & 100 \\
\hline P12 & 22 & M & -100 \\
\hline P13 & 24 & $M$ & 73 \\
\hline P14 & 22 & M & -30 \\
\hline P15 & 28 & M & 90 \\
\hline P16 & 27 & $\mathrm{~F}$ & -100 \\
\hline P17 & 21 & $\mathrm{~F}$ & -33 \\
\hline P18 & 24 & M & 46 \\
\hline P19 & 21 & $\mathrm{~F}$ & 76 \\
\hline $\mathrm{P} 20$ & 20 & $\mathrm{~F}$ & 90 \\
\hline P21 & 32 & $\mathrm{~F}$ & -20 \\
\hline P22 & 33 & $F$ & 88 \\
\hline P23 & 28 & M & 88 \\
\hline P24 & 22 & M & -30 \\
\hline P25 & 23 & $\mathrm{~F}$ & 100 \\
\hline P26 & 32 & $\mathrm{~F}$ & 90 \\
\hline P27 & 32 & $\mathrm{~F}$ & 100 \\
\hline P28 & 24 & $\mathrm{~F}$ & 100 \\
\hline P29 & 24 & M & 37 \\
\hline P30 & 31 & M & 89 \\
\hline
\end{tabular}

$\overline{a^{a} \text { Edinburgh handedness inventory (Oldfield, 1971) with score range from }-100 \text { (extremely left-handed; score }}$ below -40 left-handedness) to +100 (extremely right-handed; score above +40 right-handedness) and the score between -40 to +40 indicate ambidexterity.

\section{Materials and Methods}

Participants. Thirty healthy volunteers (13 males; mean \pm SD age $26.2 \pm$ 4.9 years) participated in one magnetic resonance imaging (MRI) and three brain stimulation sessions. We excluded participants with a previous history of neurological or psychiatric disorders and contraindications to MRI and transcranial magnetic stimulation (Rossi et al., 2009) based on a semistructured interview. Before taking part in the study, all participants provided written informed consent in accordance with protocols approved by the Central University Research Ethics Committee of the University of Oxford. The study was conducted in compliance with the latest version of the Declaration of Helsinki. We did not exclude participants based on their handedness and the Edinburgh handedness inventory (Oldfield, 1971) was used to assess hand dominance. The full score based on the Edinburgh handedness inventory was entered into the statistical analyses. Demographic data of the participants are presented in Table 1.

Visual stimuli and free visual exploration task. The stimuli consisted of 48 full-color pictures of both rural and urban landscapes (example in Fig. $1 A)$. To rule out a potential influence of lateralized features in the pictures on the symmetry of visual exploration, left-to-right mirrored versions were introduced, resulting in a total of 96 pictures. The pictures were presented in a dimly lit room on a cathode ray tube display (Sony Trinitron E530) with a resolution of $1024 \times 768$ pixels, 32-bit color depth, and a refresh rate of $85 \mathrm{~Hz}$. The pictures were presented full screen at a fixed distance of $60 \mathrm{~cm}$, the head of the participants being stabilized by a chin and head rest, generating a visual angle of $\sim 38^{\circ}$ on the horizontal dimension and $29^{\circ}$ on the vertical dimension. The stimuli were assigned to 6 blocks containing 16 pictures each (i.e., 8 original pictures and the corresponding 8 left-to-right mirrored versions). Each picture was presented for $5.5 \mathrm{~s}$. To ensure a common starting point of visual exploration for all participants, a black central fixation cross, subtending 
A

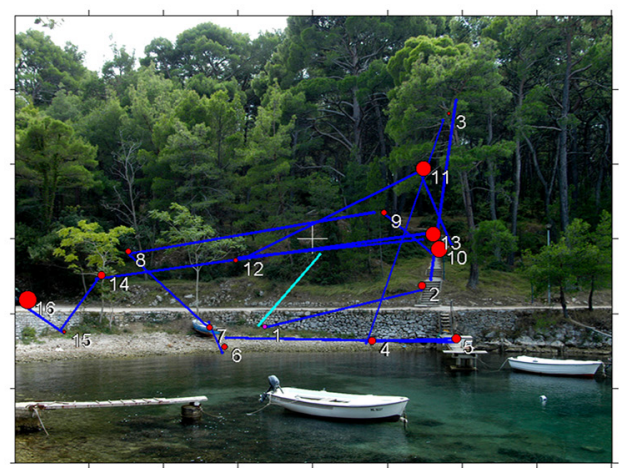

C
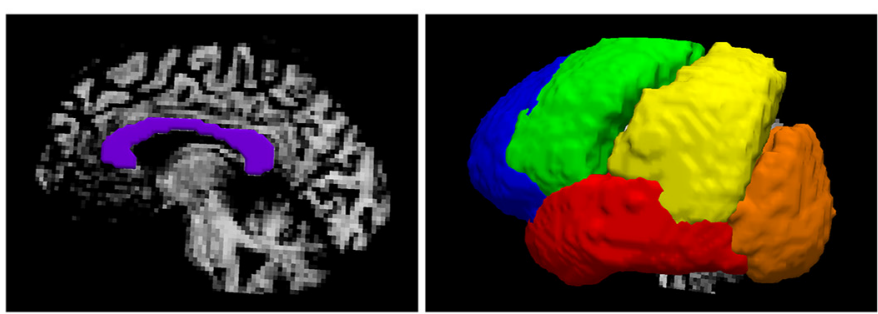

ROIs:

- CC, Corpus Callosum Mask

- OrM, Orbitofrontal Mask

FrM, Frontal Mask

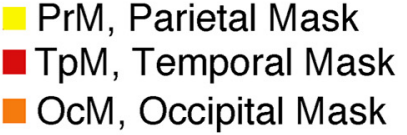

B
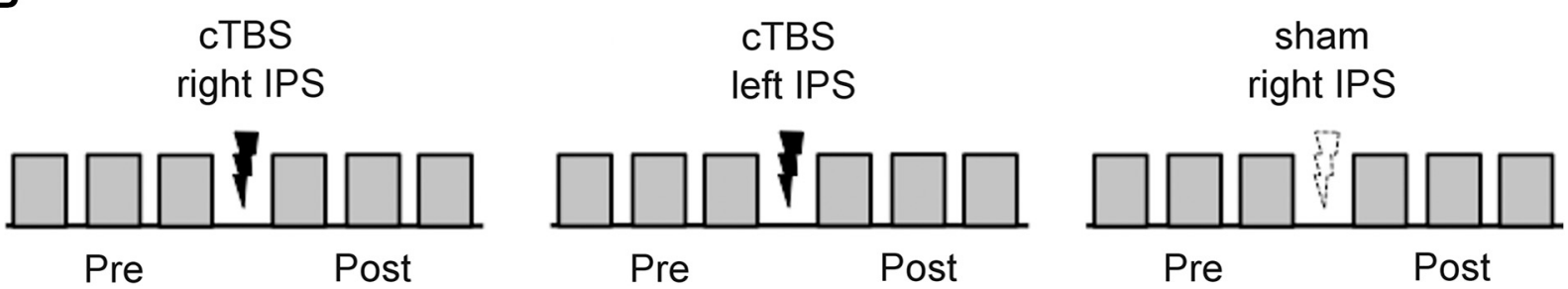

Figure 1. Study design. A, Participants performed a free visual exploration task (example of a visual picture display with a visual scanpath) while their eye movements were recorded. The cumulative fixation duration (i.e., the sum of the duration of all fixations) on the left and the right screen half was computed for each participant during each testing session at two time points (pre and post stimulation). $\boldsymbol{B}$, Schematic representation of the three testing sessions for each participant (counterbalanced order): with cTBS over the right IPS (left), with cTBS over the left IPS (central), and with sham stimulation over the right IPS (right). Gray rectangles represent blocks of the free visual exploration task before (Pre) and after (Post) stimulation application. Bolt symbols represent stimulation: real CTBS (solid symbols) and sham stimulation (dotted symbol). C, Location and delineation of regions of interest (ROIs) used in spherical deconvolution tractography (see Materials and Methods section for full details).

$1^{\circ} \times 1^{\circ}$ visual angle, was presented on a light gray background for $1.5 \mathrm{~s}$ before every picture.

Participants were asked to freely explore the pictures by means of eye movements and to accurately fixate the central fixation cross between picture presentations. The participants performed the free visual exploration task at three different test sessions, separated by at least 1 week: (1) with active stimulation over the right IPS, (2) with active stimulation over the left IPS, and (3) with sham stimulation. The order of the sessions was counterbalanced over participants. In every session, three blocks of pictures were administered as a baseline measurement (henceforth referred to as the "pre" time point). Participants then underwent brain stimulation as described below. Finally, three further blocks of pictures were administered to assess the effects of stimulation (henceforth referred to as the "post" time point). The order of the blocks and of the pictures within the blocks was randomized for every participant and testing session. A schematic representation of the experimental design is depicted in Figure 1B. During each block of visual exploration task, participants' eye movements were recorded (an example of a visual scanpath is shown in Fig. 1A).

To avoid fatigue and to allow periodic recalibration of the eye-tracking system, short breaks of $\sim 2$ min were introduced between blocks. Moreover, to allow application of stimulation, a break of $\sim 10$ min was introduced between the pre and the post time points. The duration of the pre and the post measurement time points was $\sim 11 \mathrm{~min}$ each. The whole experiment lasted between 30 and $35 \mathrm{~min}$.

Eye movements recording. Eye movements were recorded with an infrared, video-based eye-tracking system (EyeLink 1000; SR Research) with a temporal resolution of $1000 \mathrm{~Hz}$, a spatial resolution of $0.01^{\circ}$, and a gaze position accuracy of $0.25-0.5^{\circ}$ (depending on fixation accuracy during calibration). The system was calibrated by means of a $3 \times 3$ point grid before each block of the free visual exploration task and the calibration was followed up by a validation procedure. An eye movement was classed as a saccade when its velocity exceeded $35 \%$ or its acceleration exceeded $9500^{\circ} / \mathrm{s}^{2}$, and its amplitude exceeded $0.1^{\circ}$. Eye movements were parsed online into fixations and saccades and stored for offline analysis. Minimal fixation duration was set at $100 \mathrm{~ms}$.
rTMS. Continuous theta burst stimulation (cTBS), a patterned rTMS protocol, has been shown to exert strong inhibitory neural effects and, when applied over the PPC, to influence the spatial allocation of visual attention (Nyffeler et al., 2008, 2009; Cazzoli et al., 2009). In the current study, cTBS was delivered using a Magstim Rapid stimulator connected to four booster modules (Booster Module Plus), producing biphasic pulses (the most effective current induced in the brain tissue corresponding to the second half-wave of a biphasic pulse; Salvador et al., 2011). For cTBS over the right and the left IPS, the stimulator was connected to a commercially available figure-of-eight coil with a diameter of $70 \mathrm{~mm}$ (Magstim). Sham stimulation (over the right IPS) was performed with a figure-of-eight coil connected to a commercially available placebo coil system (Magstim). In the sham setup, the coil generated the same discharge noises as real stimulation and produced a mild cutaneous sensation, but did not deliver active stimulation of the underlying cortical tissue. The stimulator was driven by a laptop (Dell Latitude) running a customized script with the stimulation parameters described below and programmed in E-Prime 2.0 Pro (Psychology Software Tools).

TMS was delivered by means of a modified cTBS protocol described previously (Nyffeler et al., 2008; Cazzoli et al., 2009; Nyffeler et al., 2009; Cazzoli et al., 2012). In brief, the modified cTBS protocol comprised 801 pulses delivered in a continuous train of 267 bursts. Each burst consisted of 3 pulses at $30 \mathrm{~Hz}$, repeated at $6 \mathrm{~Hz}$. This modified cTBS protocol has been shown to exert inhibitory effects lasting at least 30 min over the PPC (Nyffeler et al., 2008) and over the primary motor cortex (Goldsworthy et al., 2012).

Coil positioning was performed by means of MRI-guided neuronavigation using a frameless stereotactic system (BrainSight; Rogue Research). An MRI anatomical scan was acquired for every participant (see below for details). The right and left IPS stimulation sites (within the posterior part of IPS; right and left IPS1/2; Silver and Kastner, 2009) were localized on the individual MRI scans of each participant using slice- and 3D-rendered information. The frameless stereotactic system was then used to coregister neuroanatomical sites with landmarks on the scalp surface. The coil was held in position by the experimenter during the duration of the cTBS train with the handle pointing posteriorly and at an 
angle of $45^{\circ}$ with respect to the participant's sagittal plane (with the present setup and coil orientation, the direction of the current induced in the brain tissue was thus posterior-anterior, as defined with respect to the first phase of the biphasic pulses and as commonly used for cTBS application; Huang et al., 2005). cTBS was delivered at $80 \%$ of the participants' individual resting motor threshold of the contralateral small hand muscles. The resting motor threshold was defined as the minimal stimulator output of TMS single pulses that was able to elicit consistent motor responses (i.e., in at least three of five consecutive trials) in the small hand muscles of the contralateral, relaxed hand.

Behavioral data analysis. The cumulative fixation duration (i.e., the sum of the duration of all fixations) on the left and the right half of the screen was computed for each participant for each stimulation condition (cTBS left IPS, cTBS right IPS, sham), and time point (pre, post). The percentage cumulative fixation duration was then calculated by dividing the cumulative fixation duration on the left and the right screen halves, respectively, by the total cumulative fixation time (i.e., the sum of the cumulative fixation time on the left and on the right screen half) and multiplying by 100 (see the formulae below).

$\%$ cumulative fixation duration on the left screen half

$$
=\left(\begin{array}{c}
\text { cumulative fixation } \\
\text { duration on the left screen half } \\
\text { cumulative fixation } \\
\text { duration on (left screen half }+ \text { right screen half) }
\end{array}\right) * 100
$$

$\%$ cumulative fixation duration on the right screen half

$$
=\left(\begin{array}{c}
\text { cumulative fixation } \\
\text { duration on the right screen half } \\
\text { cumulative fixation } \\
\text { duration on (left screen half }+ \text { right screen half) }
\end{array}\right) * 100
$$

First, the initial spatial bias (i.e., at time point pre) of each participant in each stimulation condition was assessed. The initial spatial bias was calculated by subtracting $50 \%$ from the percentage cumulative fixation duration on the right screen half as follows:

Initial spatial bias

$$
=\left[\left(\begin{array}{c}
\text { cumulative fixation } \\
\text { duration on the right screen half } \\
\begin{array}{c}
\text { cumulative fixation duration on } \\
\text { (left screen half }+ \text { right screen half })
\end{array}
\end{array}\right) * 100\right]-50
$$

Therefore, positive values indicate a rightward bias and negative values a leftward bias. To assess the consistency between the initial biases across testing sessions, we calculated an intraclass correlation coefficient and the corresponding $F$ statistic (assessing the null hypothesis of no consistency). Moreover, we compared the initial biases across testing sessions on a group level by means of a repeated-measures ANOVA (rmANOVA) with the within factor being the stimulation condition (cTBS left IPS, cTBS right IPS, sham).

To evaluate the effects of the stimulation condition on the spatial distribution of free visual exploration time at a group level, we analyzed the percentage cumulative fixation duration by means of an rmANOVA with the within factors being stimulation condition (cTBS left IPS, cTBS right IPS, sham), time point (pre, post), and screen side (left, right). Post hoc comparisons were performed by means of Bonferroni-corrected $t$ tests. To follow up on the effects on an individual level, we computed a spatial bias shift for each participant, calculated as the percentage cumulative fixation duration on the right screen half after (post) stimulation minus the percentage cumulative fixation duration on the right screen half before (pre) stimulation. Therefore, positive values indicate a rightward shift of the bias and negative values a leftward shift.

Finally, to investigate the link between the behavioral results (individual differences in response to cTBS) and the neuroimaging data (structural variability within neuronal networks), we calculated a measure, which indicates a shift in the allocation of spatial attention (the atten- tional shift) taking into account sham effects. For this purpose, we normalized spatial bias shift after cTBS over the left IPS or cTBS over the right IPS with respect to sham effects. First, the post spatial bias (i.e., at time point poststimulation) of each participant in each stimulation condition (left IPS, right IPS, and sham) was assessed. The postspatial bias was calculated in the same way as the initial spatial bias; that is, by subtracting $50 \%$ from the percentage cumulative fixation duration on the right screen half as follows:

post spatial bias

$$
=\left[\left(\begin{array}{c}
\text { cumulative fixation } \\
\text { duration on the right screen half } \\
\hline \begin{array}{c}
\text { cumulative fixation duration on } \\
\text { (left screen half }+ \text { right screen half })
\end{array}
\end{array}\right) * 100\right]-50
$$

A normalized spatial bias shift (see the formulae below) was then calculated by dividing the spatial bias shift (i.e., the difference between post spatial bias and initial spatial bias; after cTBS over the left or the right IPS, respectively) by the absolute sham effect (i.e., the absolute value of the difference between post spatial bias and initial spatial bias in the sham condition). Again, positive values indicate a rightward shift and negative values a leftward shift. Differences between the normalized spatial bias shift after cTBS over the left IPS and cTBS over the right IPS were tested by means of a paired-samples $t$ test.

normalized spatial bias shift $\mathrm{left} \mathrm{IPS} \mathrm{cTBS}$

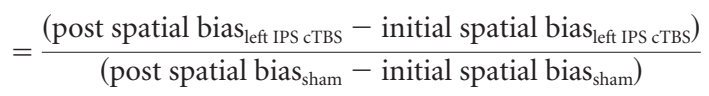

normalized spatial bias shift right IPS cTBS $_{\text {. }}$

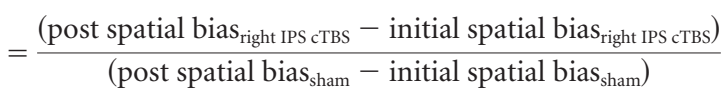

MRI data acquisition and preprocessing. T1-weighted scans were acquired at the Oxford Centre for Functional Magnetic Resonance Imaging of the Brain (FMRIB) using a 3T Verio scanner with a 32-channel head coil (Siemens). T1-weighted structural scans were acquired using magnetization-prepared rapid acquisition gradient echo sequence (MPRAGE) with the following parameters: $\mathrm{TR}=2040 \mathrm{~ms}, \mathrm{TE}=4.7 \mathrm{~ms}$, flip angle of $8^{\circ}$ and a resolution of $1 \times 1 \times 1 \mathrm{~mm}^{3}$. T1-weighted scans were used for MRI-guided neuronavigation used to target cTBS stimulation sites (see above).

Diffusion magnetic resonance imaging data were acquired using single-shot echo planar imaging (EPI) and a monopolar sequence with a voxel size of $2 \times 2 \times 2 \mathrm{~mm}^{3}$ and the following parameters: TR $=9600 \mathrm{~ms}$ and $\mathrm{TE}=87 \mathrm{~ms}$, in plane parallel imaging $(\mathrm{GRAPPA})$ factor $=2$. The diffusion weighting was isotropically distributed along 60 directions $(b=$ $1500 \mathrm{~s} / \mathrm{mm}^{2}$ ) plus 4 volumes were acquired without diffusion weighting. To improve the signal-to-noise ratio and to allow correction of geometric distortion resulting from EPI acquisition, for each participant, two sets of whole-brain diffusion-weighted data were acquired with reversed phaseencoding direction (anterior-posterior vs posterior-anterior; Chang and Fitzpatrick, 1992; Andersson et al., 2003).

Diffusion-weighted scans from all participants were preprocessed for distortion correction using the pipelines developed for the Human Connectome Project (Glasser et al., 2013; Sotiropoulos et al., 2013) and the latest FSL tools (FMRIB Centre Software Library, Oxford University; Smith et al., 2004). Briefly, pairs of phase-reversed images were used to correct for susceptibility-induced distortions (Andersson et al., 2003) using the FSL topup tool. Eddy currents and subject motion were then corrected using a generative model approach with the FSL EDDY tool (Andersson and Sotiropoulos, 2015a,b). All distortion corrections were performed in a single resampling step using spline interpolation.

Tract-based spatial statistics and ROI analyses. We used a whole-brain tract-based spatial statistics (TBSS; Smith et al., 2006) to investigate whether fractional anisotropy (FA) indexing variability in microstructural properties of WM was regionally correlated with individual differ- 
ences in the relative effects of cTBS on spatial attention. After distortion correction, FA maps were generated for each participant by fitting the diffusion tensor model (Basser et al., 1994) using the FSL Diffusion Toolbox (Smith et al., 2004). All FA maps were aligned to standard Montreal Neurological Institute (MNI) space based on nonlinear registration and a mean FA image (an average FA map) was created and skeletonized by deriving a skeleton map corresponding to the center of WM bundles $(\mathrm{FA}>0.2)$. The FA data for all participants were projected onto this mean FA skeleton.

The skeletonized FA data were then fed into a general linear model (GLM) analysis. First, we examined, across the entire group of participants, the linear relationship between FA and attentional shifts after cTBS over either the left or the right IPS, controlling for the magnitude of change induced by sham stimulation (normalized spatial bias shift). The behavioral scores entered into the analysis were demeaned. We also investigated whether structural variability, indexed by FA, within any WM region would characterize subgroups of participants, defined according to differential effects of cTBS stimulation. Specifically, we performed two analyses after dividing our participants into subgroups based on left versus right attentional shifts after cTBS over the left IPS and those with expected effects of cTBS according to Kinsbourne's (1987) model (i.e., right attentional shift after cTBS over the right IPS and left attentional shift after cTBS over the left IPS) versus all other participants ("typical" vs "atypical" responders). Although we found a large variability in the magnitude of responses (attentional shifts) to cTBS over the right IPS, the direction of the response was the same in the majority of the participants; that is, a rightward shift in attentional spatial allocation. Therefore, splitting participants into subgroups according to a leftward versus rightward response to right cTBS alone would have not been informative due to considerably uneven numbers in respective subgroups (see Fig. 3A).

Accurate statistical inference in the whole-brain TBSS analyses, including correction for multiple comparisons, was achieved by using permutation-based nonparametric inference (5000 random permutation tests) within the GLM framework (Nichols and Holmes, 2002; Winkler et al., 2014). TBSS results were considered significant at $p<$ 0.05 , corrected for multiple comparisons using the threshold-free cluster enhancement method (Smith and Nichols, 2009). The location of significant TBSS results in relation to specific WM pathways was determined based on the Johns Hopkins University (JHU) WM tractography atlas (Hua et al., 2008), the MRI Atlas of Human White Matter (Mori, 2005), and the tractography atlas of human WM tracts (Thiebaut de Schotten et al., 2011b).

TBSS analyses revealed a significant association between FA within the corpus callosum and the effects of cTBS over the left IPS. After this, we conducted additional ROI linear regression analyses to assess whether the FA within the corpus callosum predicted the effects of left IPS cTBS stimulation when controlling for age, handedness, sex, and initial attentional bias (prestimulation spatial bias $=$ initial spatial bias averaged across the three stimulation sessions). We also applied two-tailed independent samples $t$ tests to examine potential differences between subgroups of participants in mean FA within the corpus callosum. The groups were defined by whether there were left versus right attentional shifts after the left IPS cTBS and where there was an overall "typical" versus "atypical" response to cTBS according to Kinsbourne's (1977, 1987) model. All statistical analyses were performed with the SPSS 21 software. To extract FA within the corpus callosum, we created a mask in MNI standard space using the JHU WM tractography atlas (combining body, splenium, and genu; Hua et al., 2008). FA maps, transformed to MNI space, were then used to obtain the mean FA value within the corpus callosum for every participant. The extracted FA values then were entered into the statistical analyses in SPSS, as described above.

Spherical deconvolution and corpus callosum tractography. We extended our TBSS analyses of the FA data by using different structural metrics of WM derived from tractography reconstructions of the corpus callosum. Spherical deconvolution based on the damped Richardson-Lucy algorithm using previously optimized parameters (Dell'acqua et al., 2010, 2013) was performed to estimate voxelwise fiber orientation distribution functions. This was followed by whole-brain tractography reconstructions with the StarTrack software (www.natbrainlab.com). The stream- lines were propagated using an Euler integration algorithm with a step size of $0.5 \mathrm{~mm}$ and an angular threshold of $45^{\circ}$. Finally, we applied a single ROI approach to execute virtual dissections of the corpus callosum using TrackVis (Ruopeng Wang, Van J. Wedeen, TrackVis.org, Martinos Center for Biomedical Imaging, Massachusetts General Hospital). For each participant, a sagittal ROI was delineated around the WM of the corpus callosum on three separate slices, one midsagittal, one situated two slices to the left, and one two slices to the right from the midslice (Thiebaut de Schotten et al., 2011b; Thiebaut de Schotten et al., 2012; Rojkova et al., 2015; Fig. 1C). The sagittal ROI was used as a seed for tractography to isolate the corpus callosum; we kept all of the streamlines that went through this sagittal ROI.

Anatomically, the corpus callosum is subdivided in the midsagittal plane into rostrum, genu, body, isthmus, and splenium, although some schemes divide the body into further subsections (Crosby, 1962; Witelson, 1989). Alternatively, the corpus callosum can be subdivided based on tractography and cortical segmentation; that is, segments corresponding to interhemispheric connections within distinct cortical lobes (Zarei et al., 2006; Hofer and Frahm, 2006; Park et al., 2008). Previous work suggests that interhemispheric connections relevant to visuospatial attention are located within the posterior portion of the corpus callosum containing fibers linking homologous regions within the parietal, temporal, and occipital lobes (Koch et al., 2011; Lunven et al., 2015). It has been also proposed that interhemispheric dynamics in visuospatial attention differ between the frontal lobes and parietal/visual cortical areas (Duecker et al., 2013; Duecker and Sack, 2015). To investigate whether there are any regionally specific differences in the microstructure and macrostructure of the corpus callosum in predicting the response to cTBS, we performed tractography-based callosal parcellation. We separated the corpus callosum fibers connecting homologous cortical areas based on cortical divisions using five target (seed) masks and a two-ROI approach as described previously (Rojkova et al., 2015). The cortical target masks were defined for orbitofrontal cortex (orbitofrontal mask), prefrontal/frontal cortex (excluding premotor and motor cortical areas; the attention network specific frontal mask), the inferior plus superior parietal lobules (the dorsal attention network specific parietal mask), the temporal cortex (temporal mask), and the occipital cortex (occipital mask; Fig. 1C; for similar approach, see Zarei et al., 2006; Hofer and Frahm, 2006). To isolate the specific parts of the corpus callosum, we retained the streamlines that went through a sagittal ROI delineated around the WM of the corpus callosum (as described above) and one of the cortical target masks (Fig. 1C). The different cortical masks were created based on the MNI structural atlas and HarvardOxford cortical atlas (Collins et al., 1995; Mazziotta et al., 2001; Desikan et al., 2006) implemented in FSL and transformed into each participant's native diffusion space using nonlinear transforms created in TBSS processing steps. After virtual dissections, for all participants, we extracted the hindrance-modulated orientational anisotropy (HMOA) and the volume of the reconstructed full corpus callosum and the five reconstructed parts of the corpus callsoum. HMOA provides information about diffusion properties of the WM specific to the diffusion orientation and, within regions of crossing fibers, this measure provides a better estimate of the microstructural organization of the WM than the FA (Dell'acqua et al., 2013; Rojkova et al., 2015). The tract volume was calculated from the number of voxels intersected by the streamlines of each tract, which provides a macrostructural measure of the space occupied by the reconstructed pathway. Because tractography was performed in the native space to control for variability in brain/hemisphere size (which could affect the volume of the reconstructed tracts, i.e., larger brain = larger tracts), for each participant, we normalized the tract volume by the hemisphere WM volume (tract volume/total WM volume). We used the Shapiro-Wilk tests (Shapiro and Wilk, 1965) to examine Gaussian distribution of the tractography data and a normal distribution of HMOA and volume measures was confirmed.

To determine whether the HMOA index within the corpus callosum or the corpus callosum volume would predict the effects of cTBS over either the left or the right IPS (normalized spatial bias shift taking into account the magnitude of change induced by sham stimulation; the dependent variable), we performed linear regression analyses controlling 
A

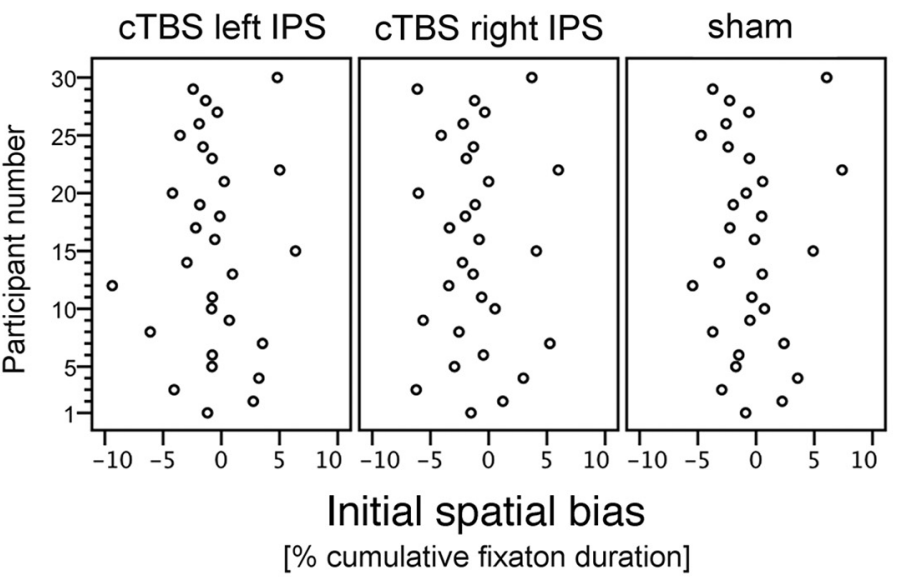

B

[\% cumulative fixaton duration]
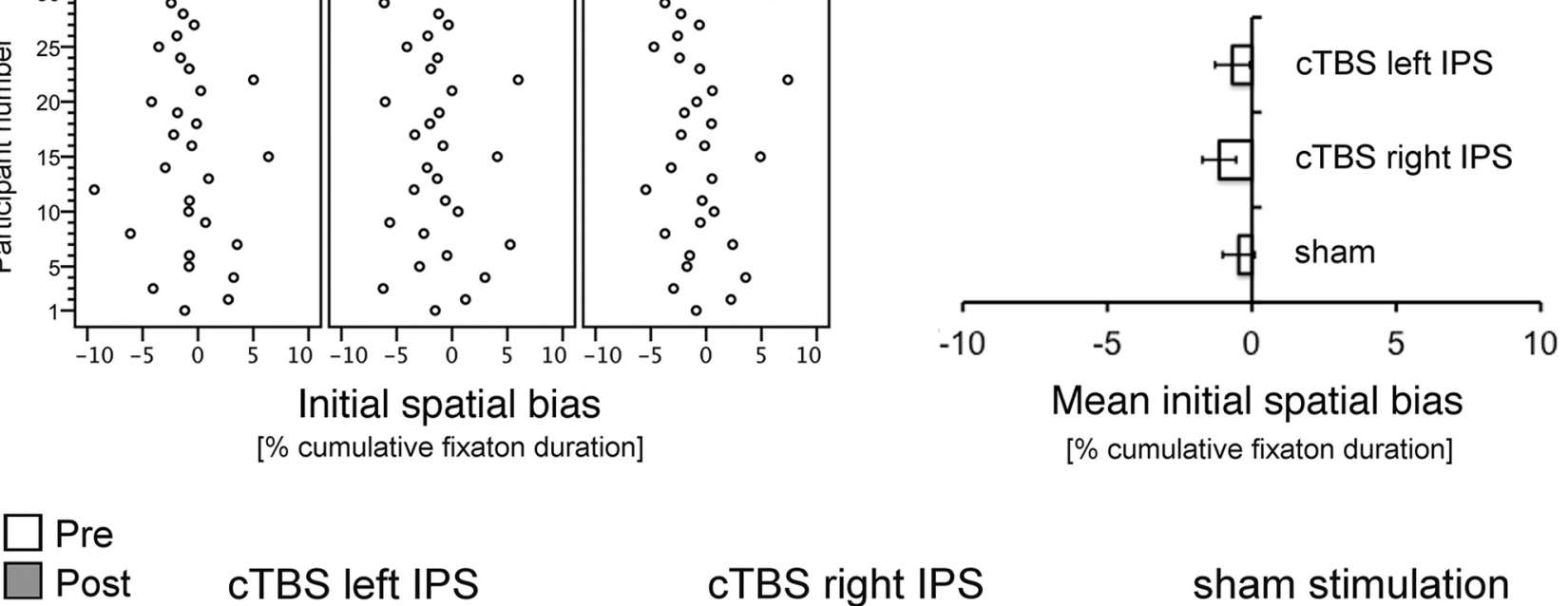

cTBS left IPS

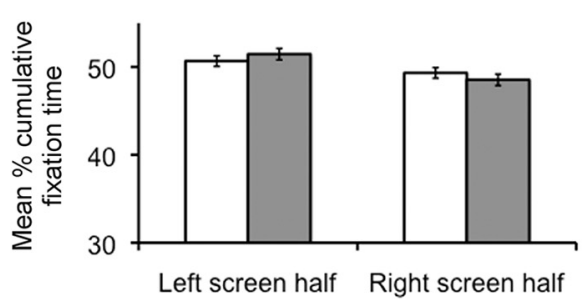

cTBS right IPS

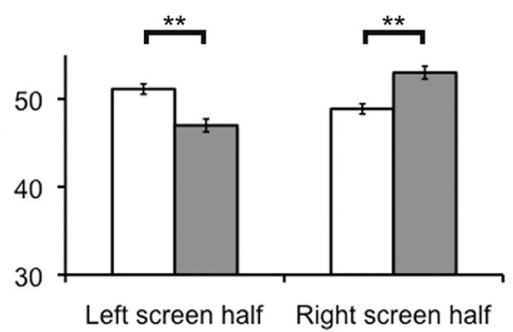

sham stimulation

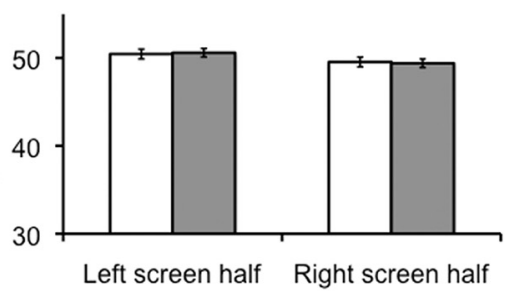

Figure 2. $\quad \boldsymbol{A}$, Initial spatial bias (i.e., at time point pre; percentage cumulative fixation duration) across testing sessions in individual participants (left) and at a group level (right). Negative values indicate a leftward bias; positive values indicate a rightward bias. Bars indicate the SEM. $\boldsymbol{B}$, Mean percentage cumulative fixation time at a group level on the right and the left screen halves, pre and post stimulation, in the condition with cTBS over the left IPS (left), CTBS over the right IPS (central), and sham stimulation over the right IPS (right). Error bars indicate SEM. Asterisks denote significant post hoc tests ( $\left.{ }^{* *} p<0.01\right)$.

for age, handedness, sex, and initial attentional bias (initial spatial bias averaged across the three stimulation sessions). The secondary analyses addressed the correlation between the measures of structural variability within the corpus callosum, identified as predictors of individual differences in response to cTBS over either the left or the right IPS. To correct for multiple comparisons in the regression and correlation analyses, we applied a false discovery rate (FDR) correction based on the BenjaminiHochberg FDR method, as implemented in the MATLAB Bioinformatics toolbox (Benjamini and Hochberg, 1995; Matlab R2012a; The MathWorks). Finally, we applied two-tailed independent-samples $t$ tests to assess potential differences between subgroups of participants (defined by the left vs right attentional shift after cTBS over the left IPS; or the overall "typical" vs "atypical" response to cTBS according to Kinsbourne's model) in the structural variability (HMOA index and volume) of the corpus callosum. All statistical analyses were performed with the SPSS 21 software.

\section{Results}

\section{Behavioral data: effects of cTBS}

As shown in Figure $2 A$, left, the initial spatial bias showed substantial interindividual variability in its direction (i.e., some participants had a leftward and some a rightward bias) and its magnitude. However, the direction of the initial spatial bias was relatively constant within participants across testing sessions (i.e., there was low intraindividual variability): 25 of 30 participants $(83.3 \%)$ had the same direction of initial spatial bias (leftward or rightward) in all three testing sessions and the remaining 5 participants $(16.7 \%)$ had the same direction of spatial bias in 2 of 3 testing sessions. This was also reflected in an intraclass correlation coefficient of 0.829 ( $p<0.001)$, indicating substantial con- sistency (Landis and Koch, 1977a,b) for the initial biases across testing sessions. Moreover, the rmANOVA indicated that, on a group level (Fig. $2 A$, right), the initial spatial bias was not significantly different between testing sessions $\left(F_{(2,58)}=2.128, p=\right.$ $0.128)$.

The rmANOVA on the spatial distribution of free visual exploration time revealed a significant three-way interaction between the factors stimulation condition, time point, and side $\left(F_{(2,58)}=25.237, p<0.001\right)$. As confirmed by post hoc tests, on a group level, cTBS over the right IPS triggered a significant rightward shift (i.e., there was a reduced percentage cumulative fixation time on the left screen half and an increased percentage cumulative fixation time on the right screen half); in contrast, cTBS over the left IPS and sham stimulation over the right IPS did not have any significant effect (Fig. $2 B$ ).

On an individual level (Fig. 3A), the spatial bias shift triggered by cTBS over the right IPS showed conspicuous interindividual variability in its magnitude (i.e., some individuals showed clearly greater shifts than others). However, the direction of the spatial bias shift had low interindividual variability: 27 of 30 participants (90\%) showed a rightward bias shift after cTBS over the right IPS and only $3(10 \%)$ showed a leftward bias shift. In contrast to this, the spatial bias shift triggered by cTBS over the left IPS showed striking interindividual variability both in its magnitude and its direction: 18 of 30 participants (60\%) showed a leftward bias shift and 12 of 30 participants (40\%) showed a rightward bias shift.

The analysis of the mean normalized spatial bias shift revealed a significant difference between the cTBS left IPS and the cTBS 

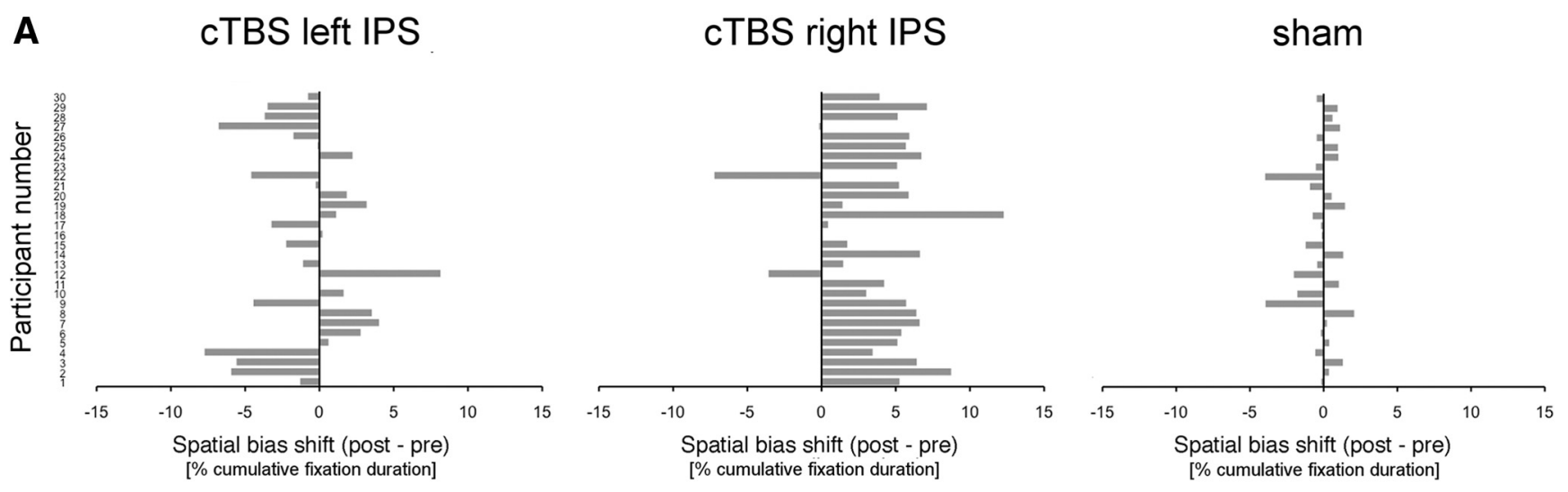

B

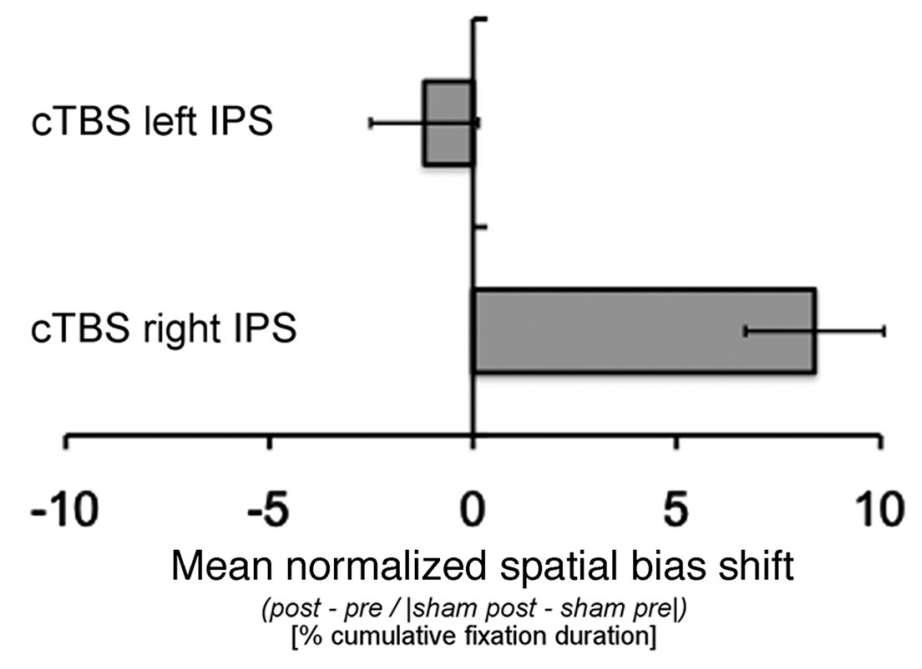

Figure 3. A, Individual bias spatial shifts (post - pre percentage cumulative fixation durations on the right screen half) after cTBS over the left IPS (left), after cTBS over the right IPS (central), and after sham stimulation (right). Positive values indicate a rightward shift; negative values indicate a leftward shift. $\boldsymbol{B}$, Mean normalized spatial bias shift (calculated as follows: post minus pre spatial bias shift divided by the absolute value of the difference between post minus pre sham condition) after cTBS over the left IPS and cTBS over the right IPS. Positive values indicate a rightward shift; negative values indicate a leftward shift. Error bars indicate SEM.

right IPS condition $\left(t_{(29)}=5.0, p<0.001,2\right.$-tailed). Whereas cTBS over the left IPS triggered a modest leftward shift, cTBS over the right IPS triggered a more conspicuous rightward shift (Fig. 3B).

\section{Neuroimaging data: structural variability as a predictor of attentional shifts after cTBS}

We first performed whole-brain TBSS analyses to determine whether structural variability within the WM as indexed by FA indicates the anatomical basis of individual differences in response to cTBS over the left and right IPS. These analyses demonstrated a strong linear association between FA values within the corpus callosum and attentional shifts after cTBS over the left IPS (Fig. 4A). Specifically, we found the FA to be higher in the corpus callosum of participants with stronger leftward shifts in allocation of attention after cTBS over the left IPS. The higher FA was detected within both anterior and posterior parts of the corpus callosum, as well as throughout the entire body. There were no significant associations between variability in the FA and the individual differences in the response to cTBS over the right IPS.

Our participants were not recruited based on handedness and our sample consisted of 20 right-handed, four left-handed, and six ambidextrous participants based on the Edinburgh handed- ness inventory (Oldfield, 1971). Some previous studies have suggested a link between hand preference and the morphology of the corpus callosum (Witelson, 1985, 1989; Denenberg et al., 1991; Habib et al., 1991; Tuncer et al., 2005; Josse et al., 2008; Luders et al., 2010). These reports argue that the corpus callosum is larger in left-handers and in individuals with nonconsistent right-handedness. Other researchers have not found this association (Kertesz et al., 1987; Jäncke et al., 1997; Preuss et al., 2002; Luders et al., 2003; Anstey et al., 2007). Although some studies have also indicated a potential link among handedness, brain lateralization, and hemispheric dominance in attention, this link is neither straightforward nor confirmed by all reported data and there is no substantial evidence to argue that the degree of handedness relates to spatial bias (Bryden et al., 1983; Szaflarski et al., 2002; Flöel et al., 2005a,b; Whitehouse et al., 2009; Cai et al., 2013; Szczepanski et al., 2013; Mazoyer et al., 2014; Chechlacz et al., 2015; Petit et al., 2015; Somers et al., 2015; for a recent comprehensive review see Willems et al., 2014). Nevertheless, taking all into consideration, we repeated the above TBSS analyses after covarying out handedness (continuous measure). The results were almost identical to those reported above, and indicated again a significant association between structural variability within the corpus callosum and individual differences in the response to cTBS over the left IPS (and no significant effects of 

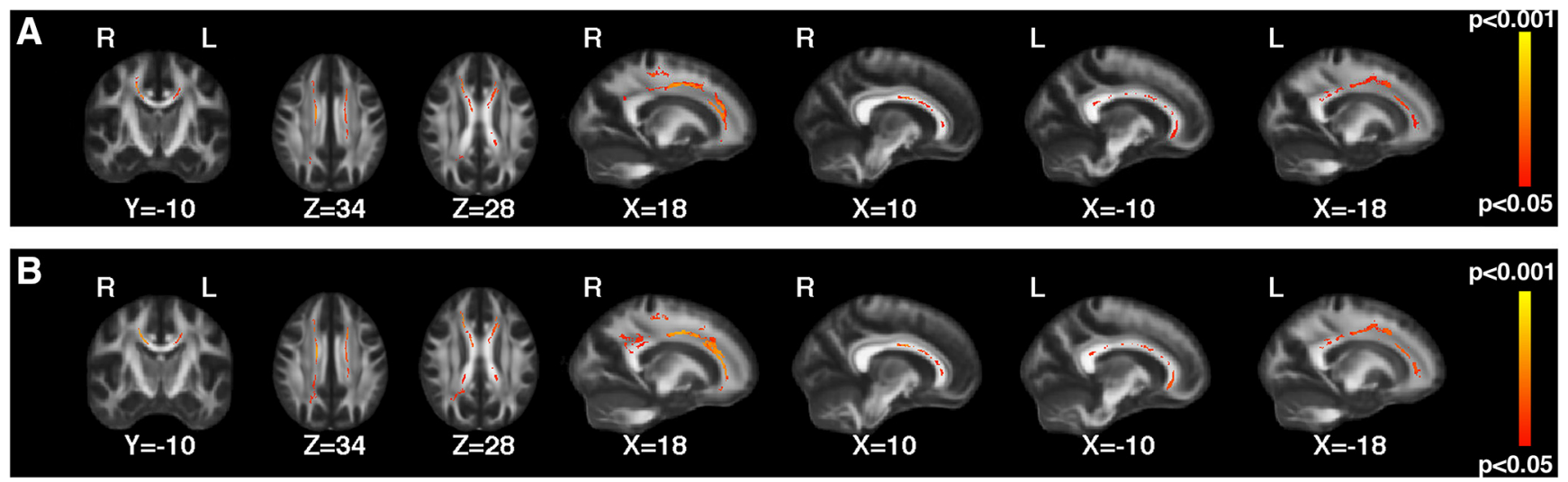

Figure 4. Results of TBSS analyses. $\boldsymbol{A}$, Higher FA in the corpus callosum was associated with stronger leftward shifts in attention after stimulation over the left IPS. $\boldsymbol{B}$, Higher FA in the corpus callosum was associated with stronger leftward shifts in attention after cTBS over the left IPS after controlling for the effect of handedness (continuous variable). All results are presented after correction for multiple comparisons $(p<0.05)$. Results are displayed on the FSL FA template in the standard MNI space with given $X, Y$, and $Z$ coordinates. L, Left; $R$, right. Only parts of the TBSS skeleton where significant effects $(p<0.05)$ were found are shown. The colors depict $p$-values.
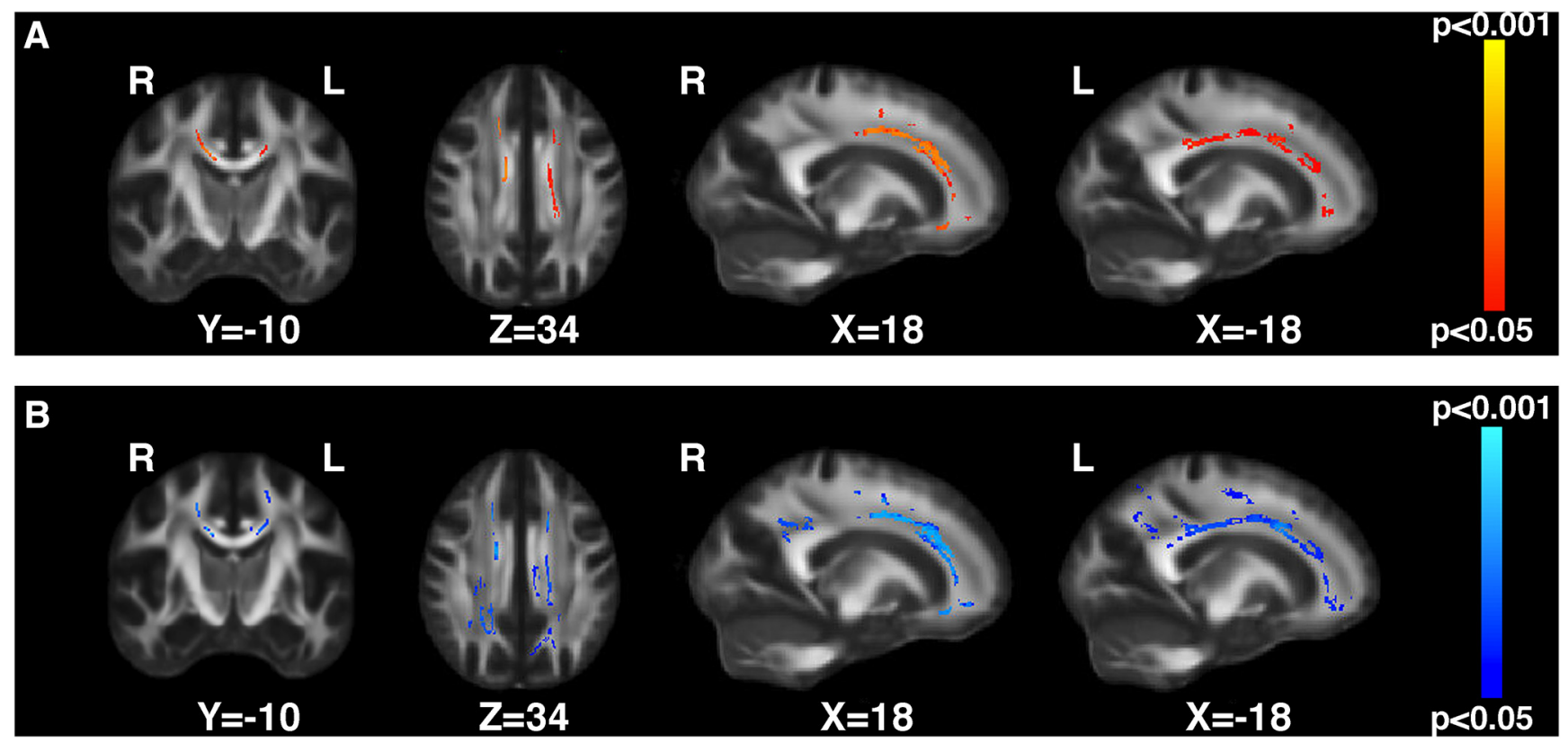

Figure 5. Results of TBSS group analyses. Higher FA in the corpus callosum in participants with leftward versus rightward attentional shifts after cTBS over the left IPS (presented in red-yellow; A) and with "typical" versus "atypical" overall responses to cTBS as predicted by Kinsbourne's $(1987,1993)$ model (i.e., a rightward attentional shift after cTBS over the right IPS cTBS, and a leftward attentional shift after cTBS over the left IPS; presented in blue-light blue; $\boldsymbol{B})$. All results are presented after correction for multiple comparisons $(p<0.05)$. The results are displayed on the FSL FA template in the standard MNI space with given $X, Y$, and $Z$ coordinates. L, Left; $R$, right. Only parts of the TBSS skeleton where significant effects $(p<0.05)$ were found are shown. The colors depict $p$-values.

cTBS over the right IPS; Fig. 4B). Furthermore, a subsequent linear regression analysis, based on the extracted mean FA within the corpus callosum and attentional shifts after cTBS, demonstrated that FA within the corpus callosum was a significant predictor of the response to cTBS over the left IPS ( $\beta=-0.487, p=0.01)$ but not over the right IPS $(\beta=$ $-0.107, p=0.59)$, after controlling for the effects of age, handedness, gender, and initial spatial bias (prestimulation attentional bias averaged across the three stimulation sessions). Therefore, importantly, the link between mean FA within corpus callosum and response to cTBS over the left IPS emerged to be unrelated to initial spatial bias, degree of handedness, age, and sex $(p>0.5)$.

We next investigated whether structural variability, indexed by FA, within any WM region would characterize participants subgrouped by the effects of cTBS stimulation. The whole-brain voxelwise TBSS analyses demonstrated significant differences in the FA within the corpus callosum when comparing participants with left $(n=18)$ versus right $(n=12)$ attentional shifts after cTBS over the left IPS (Fig. 5A) and when comparing participants with responses to cTBS as predicted by Kinsbourne's (1977, 1987) model (i.e., those showing rightward attentional shifts after cTBS over the right IPS and leftward attentional shifts after cTBS over the left IPS vs all other participants; "typical" versus "atypical" responders; $n=16$ versus 14 participants, respectively; Fig. $5 B)$. Higher FA was found in the anterior and midbody part of the corpus callosum in participants with left versus right attentional shifts after cTBS over the left IPS; for the contrast between "typical" and "atypical" responders, differences in FA were found across the entire length of the corpus callosum. Additional ROI 
analyses based on the extracted mean FA values within the corpus callosum confirmed that there was higher FA in participants with a left shift in the allocation of visual attention after cTBS over the left IPS $\left(t_{(28)}=2.43 ; p=0.022\right)$ and in those with "typical" response to cTBS $\left(t_{(28)}=2.97 ; p=0.006\right)$.

The whole-brain tract-based spatial statistics analyses indicated that high FA within the corpus callosum was the sole significant predictor of the effects of cTBS. Although in the current study, we did not find any significant link between responses to cTBS stimulation and intrahemispheric connections (i.e., frontoparietal WM pathways), the potential role of these pathways should not be overlooked. These pathways have been linked to individual differences in spatial bias (Thiebaut de Schotten et al., 2011a; Chechlacz et al., 2015), likely mediate compensatory changes in regional cortical activity within each hemisphere triggered by cTBS (Sack et al., 2007; Plow et al., 2014; Marshall et al., 2015), and contribute to the differential activation of distant regions within the attentional networks of the left versus the right hemispheres in response to stimulation. Strikingly, one recent study found a link between individual differences in TMSinduced modulation of performance in spatiotemporal visual detection task and structural variability in intrahemispheric frontoparietal pathways (Quentin et al., 2015).

The corpus callosum is located within brain regions that have a complex WM organization (i.e., with high WM density and multiple crossing pathways; Thiebaut de Schotten et al., 2011b). FA measures based on traditional diffusion tensor models may fail to depict tissue microstructure precisely within pathways affected by crossing fibers, whereas indices calculated using methods able to resolve crossing fibers give better estimates in such cases (Jones et al., 2013; Dell'acqua et al., 2013; Rojkova et al., 2015). Spherical deconvolution based on estimating a distribution of fiber orientations from the diffusion signal allows a more accurate characterization of WM properties in regions where there are crossing fibers, overcoming some of the limitations of traditional diffusion tensor methods (Dell'acqua et al., 2013). To verify our results from the tensor model and TBSS analyses, we performed tractography of the corpus callosum and then calculated the tract volume and the spherical deconvolution-derived HMOA index for the different parts of the corpus callosum. It should be noted here that, although the FA and HMOA indexes are calculated based on different approaches to modeling diffusion data, both measures are sensitive to microstructural organization of WM such as myelination, axon density, axon diameter, and fiber dispersion (Dell'acqua et al., 2013), whereas volume is calculated based on the space occupied by the tractography reconstructed WM tract and denotes its size. Tractography allowed us to extend our analyses by examining the link between variability in both the microstructure (FA and HMOA indices) and macrostructure (tract volume) of the corpus callosum in relation to the responses to cTBS, as well as to test for any regional specificity in the link between the structural organization of the corpus callosum and individual differences in response to stimulation.

Linear regression analyses demonstrated that the HMOA index was a significant predictor of leftward attention shifts after cTBS over the left IPS (across the entire corpus callosum, $\beta=-0.523, p=0.028$ FDR corrected, orbitofrontal part $\beta=$ $-0.560, p=0.020$ FDR corrected, parietal part $\beta=-0.515, p=$ 0.035 FDR corrected and temporal part $\beta=-0.503, p=0.035$ FDR corrected but not in the case of either the prefrontal/frontal or occipital parts $p>0.5$ ) after controlling for the effects of age, handedness, sex, and spatial bias. Supplementary correlation analyses indicated a strong association between the HMOA index and the response to cTBS over the left IPS. Specifically, a higher mean HMOA index within the entire corpus callosum was associated with a larger leftward shift in the allocation of visual attention after cTBS over the left IPS $(r=-0.46 ; p=0.034$ FDR corrected), whereas it was not related to attentional shifts after cTBS over the right IPS ( $p>0.5)$. Interestingly, we again found regional specificity in the link between a larger leftward attentional shift after cTBS over the left IPS and a higher HMOA index (i.e., there was a significant negative correlation for the orbitofrontal $(r=-0.46 ; p=0.030$ FDR corrected), parietal $(r=$ $-0.48 ; p=0.030$ FDR corrected $)$, and temporal $(r=-0.35 ; p=$ 0.028 ; although this result did not survive correction for multiple comparisons) portions of the callosal fibers (Fig. 6B). Based on both regression and correlation analyses, the effects of the volume of the corpus callosum on the response to cTBS over the left IPS were not significant $(p>0.5)$. In contrast, the effects of cTBS over the right IPS could be predicted by the volume of the entire corpus callosum $(\beta=-0.417, p=0.037$; although this result did not survive correction for multiple comparisons), but not by the HMOA index $(p>0.5)$. We did not find any regional specificity in the link between response to cTBS over the right IPS and the volume of the corpus callosum $(p>0.5)$. Subsequent correlation analysis demonstrated a negative association between the effect of cTBS over the right IPS and the volume of the entire corpus callosum (Fig. 6C; smaller volume of corpus callosum corresponding to larger right attention shift after cTBS over the right IPS; $r=-0.31 ; p=0.047$; although this result did not survive correction for multiple comparisons).

Two earlier studies demonstrated that interhemispheric connections relevant to visuospatial attention are located within the posterior portion of the corpus callosum (Koch et al., 2011; Lunven et al., 2015). In addition, a recently proposed hybrid model of attention control suggests that there may be striking regional differences (between frontal, parietal and occipital areas) in hemispheric asymmetries and right hemisphere dominance (Duecker and Sack, 2015). In contrast, our analysis found the association between microstructural variability and response to cTBS stimulation within both the anterior and posterior corpus callosum (i.e., the orbitofrontal, parietal and temporal parts of the corpus callosum). Although the findings concerning the orbitofrontal part of the corpus callosum are somewhat surprising, there is a growing body of evidence that anterior prefrontal cortex (equivalent to the orbitofrontal mask used here) plays a role in attentional control in visual search (for a review see Pollmann, 2004, 2012). There was also a link between microstructural variability of the entire corpus callosum and the response to cTBS stimulation. Finally, we report no regional specificity in the link between macrostructural variability within the corpus callosum and the response to cTBS stimulation. Therefore, overall, our data support Hilgetag's (2001) proposal that, in addition to the parietal cortex, other cortical areas within the attention networks may interact competitively and they also demonstrate that these interactions are mediated by the corpus callosum.

Together, the results provided by the regression and correlation analyses demonstrated that the HMOA index is a predictor for leftward attentional shifts after cTBS over the left IPS (higher HMOA index predicts both the leftward direction and the extent of the attentional shift), whereas the volume of the corpus callosum predicts rightward attentional shifts after cTBS over the right IPS (smaller volume predicts greater rightward shift). Therefore, we conducted a final supplementary group analysis, which confirmed that there was a significantly higher HMOA index within the corpus callosum $\left(t_{(28)}=2.61 ; p=0.013\right)$ and a lower corpus 
A
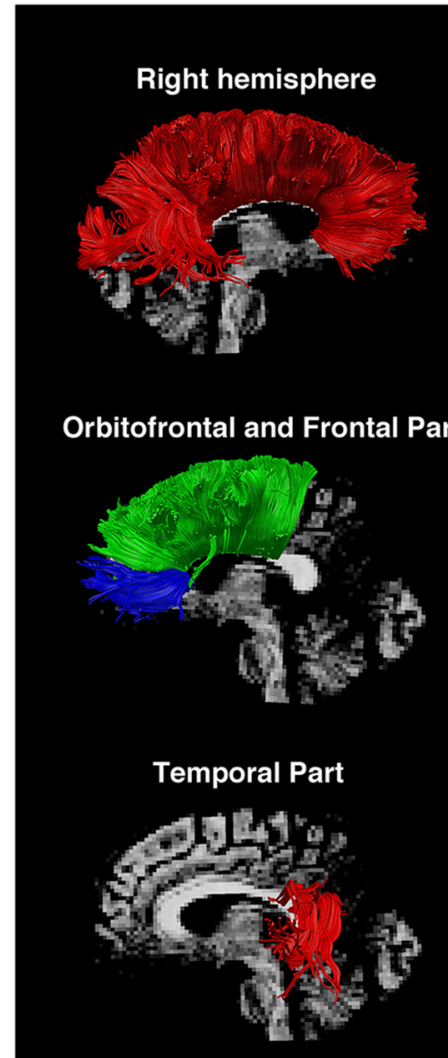

C

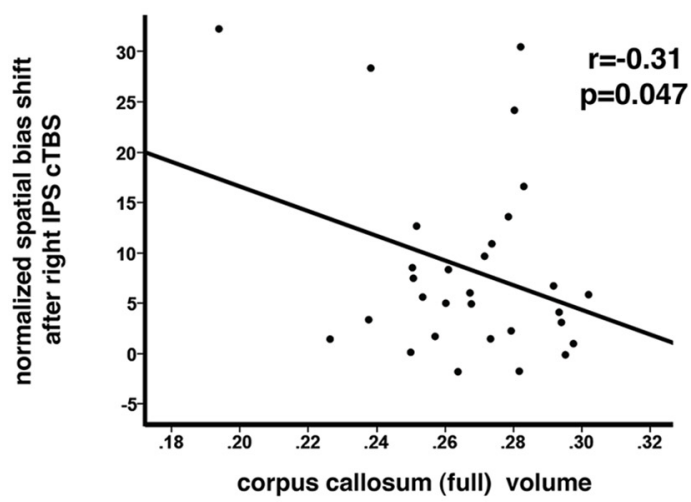

B
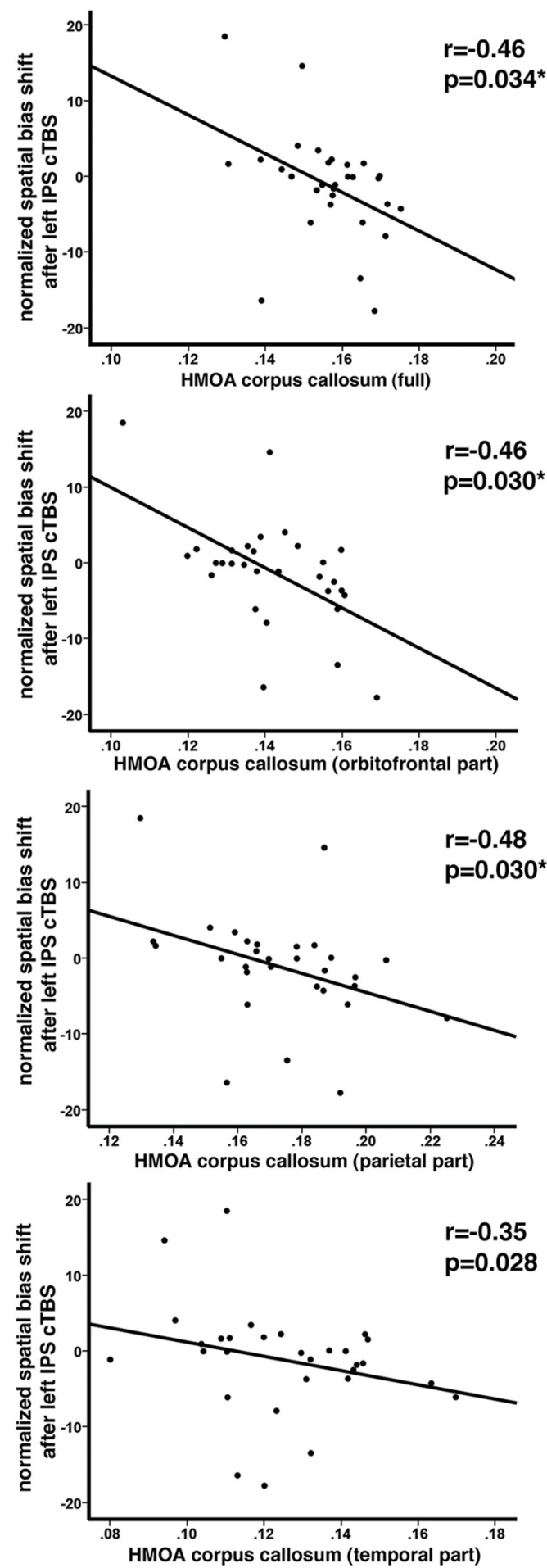

Figure 6. Results of spherical deconvolution tractography. $A$, Examples of spherical deconvolution tractography reconstruction of the entire corpus callosum (top) and of different callosal parts: orbitofrontal (blue), prefrontal/frontal (green), parietal (yellow), temporal (red), and occipital (orange). B, Correlations between HMOA measures within the corpus callosum and the effect of cTBS over the left IPS (i.e., normalized shift in the allocation of spatial attention/normalized spatial bias shift). C, Correlations between normalized volume of the corpus callosum and the effect of cTBS over the right IPS (i.e., normalized shift in the allocation of spatial attention/normalized spatial bias shift). Negative values indicate leftward and positive rightward shift in the allocation of spatial attention after cTBS. * ${ }^{*}$ Correlation is significant after correction for multiple comparisons (FDR-corrected $p$-values).

callosum volume $\left(t_{(28)}=-2.42 ; p=0.024\right)$ in participants with "typical" versus "atypical" responses as predicted by Kinsbourne's $(1987,1993)$ model. It should be noted that we found no correlation between the HMOA index and the volume of the entire corpus callosum ( $p>0.5)$. However, interestingly, we found a significant negative correlation between the HMOA in- dex and the volume of the parietal portion of the corpus callosum interconnecting areas within inferior and superior parietal lobule $(r=-0.48 ; p=0.015$ FDR corrected). Although we only observed a weak correlation between attentional shifts after cTBS over the right IPS and the size of the corpus callosum (Fig. 6C), the following findings (as presented above) further support the 
association between the volume of the corpus callosum and the effect of cTBS stimulation: (1) significant group differences in the size of the corpus callosum between participants with "typical" (i.e., rightward attentional shifts after cTBS over the right IPS and leftward attentional shifts after cTBS over the left IPS) versus "atypical" responses to cTBS as predicted by Kinsbourne's model and (2) a negative correlation between the HMOA index and the volume within the specific portion of the corpus callosum connecting parietal attention networks.

Although some previous reports argued that the corpus callosum is larger in left-handers and in individuals with nonconsistent right-handedness (Witelson, 1985, 1989; Denenberg et al., 1991; Habib et al., 1991; Tuncer et al., 2005; Josse et al., 2008; Luders et al., 2010; although other reports found so such link Kertesz et al., 1987; Jäncke et al., 1997; Preuss et al., 2002; Luders et al., 2003; Anstey et al., 2007), our additional analyses indicated that, in our group of participants, there were no associations between the overall size (volume) of the corpus callosum and handedness $(p>0.5)$. Similarly, we found no link between the volume of the corpus callosum and sex $(p>0.5$; some earlier reports argue a link between sex and the size of the corpus callosum, whereas others found no supporting evidence; Allen et al., 1991; Bishop and Wahlsten, 1997; Dubb et al., 2003; Ardekani et al., 2013). It should be noted, however, that our sample might be too small to detect such subtle anatomical differences.

Finally, there were no statistically significant correlations $(p>$ 0.5) among the FA, the HMOA index, and the volume of the corpus callosum and participants' initial spatial bias. Earlier studies suggest that individual differences in spatial biases are causally link to structural variability within the frontoparietal WM pathways (Thiebaut de Schotten et al., 2011a; Chechlacz et al., 2015). Together, previous reports and current data suggest that, whereas the structural organization of intrahemispheric connections (i.e., the frontoparietal WM pathways) mediate spatial bias, the structural organization of interhemispheric connection (i.e., the corpus callosum) mediates dynamic cross-hemispheric interactions and thus contributes differentially to hemispheric lateralization in spatial attention.

\section{Discussion}

We used an inhibitory cTBS protocol to disrupt the allocation of visuospatial attention and combined this with diffusionimaging-based assessment of structural variability in WM organization. Our data provide strong evidence that structural variability within the corpus callosum predicts both the direction and extent of attentional shifts after cTBS applied over the IPS.

Previous studies have reported strong effects of inhibitory stimulation applied over the right PPC, but no effects of stimulation over the left PPC, suggesting a clear functional asymmetry between the right and left hemispheres in attentional control (Fierro et al., 2000; Hilgetag et al., 2001; Bjoertomt et al., 2002; Hung et al., 2005; but see Dambeck et al., 2006; Sack et al., 2007; Battelli et al., 2009; Cazzoli et al., 2009; Szczepanski and Kastner, 2013). These differential effects of right versus left PPC stimulation tie in with Kinsbourne's interhemispheric rivalry model in which the spatial distribution of attention is determined by dynamic competition between the left and right hemispheres, each directing attention toward the contralateral visual field, but with a stronger contralateral bias of the right hemisphere (Kinsbourne 1977). On the group level, our findings are consistent with this: cTBS over the right IPS triggered a significant rightward shift in the distribution of fixations, whereas cTBS over the left IPS had no significant effect. However, on an individual level, cTBS over the left IPS triggered opposite responses, with some participants responding with rightward and some with leftward shifts in attention. These findings support the notion that interindividual anatomical and functional differences need to be taken into consideration to understand mechanisms of human attention (Thiebaut de Schotten et al., 2011a; Szczepanski and Kastner, 2013; Chechlacz et al., 2015).

Our whole-brain analyses showed that the structural organization of the corpus callosum was the sole significant predictor of the effects of cTBS on the allocation of spatial attention. Prior evidence suggests that variability in the structural architecture of intrahemispheric frontoparietal pathways contribute to individual differences in spatial attention (Thiebaut de Schotten et al., 2011a; Chechlacz et al., 2015). Our results extend previous findings by showing that structural variability within the corpus callosum contributes to individual differences in the effects of rTMS on allocation of spatial attention. Because rTMS can be used as a clinical tool to ameliorate neglect symptoms (Cazzoli et al., 2010), our findings have broader implications. Indeed, Lunven et al. (2015) have demonstrated that the degree of WM integrity within the corpus callosum predicts the potential for recovery from neglect (see also Carter et al., 2010; Corbetta et al., 2015).

\section{Novel dual account of callosal function in spatial attention}

To date, it is not clear how the corpus callosum regulates the interactions between the left and the right hemispheres and two opposing models-one based on interhemispheric inhibition and one on excitatory function-have been proposed (Bloom and Hynd, 2005; van der Knaap and van der Ham, 2011). The inhibitory model states that the corpus callosum maintains hemispheric lateralization, with greater interhemispheric connectivity corresponding to stronger inhibition of the nondominant hemisphere. This model matches directly Kinsbourne's proposal of hemispheric rivalry and cross-hemispheric inhibition in spatial attention (Kinsbourne, 1977). In contrast, the excitatory model argues that the corpus callosum reinforces information transfer between the hemispheres, with stronger connectivity corresponding to a greater rebalancing of activation across both hemispheres, which in turn decreases hemispheric asymmetries (Bloom and Hynd, 2005). Previous studies provide conflicting evidence that supports either the inhibitory or the excitatory model, raising the possibility that the corpus callosum plays both inhibitory and excitatory roles in interhemispheric communication. Based on our data, we put forward a dual-process model consisting of both inhibitory and excitatory mechanisms of corpus callosum function in maintaining right hemispheric dominance in spatial attention. Furthermore, we suggest that the microstructural and macrostructural organization of the corpus callosum may modulate differentially interhemispheric communication and the distribution of processing resources between the two hemispheres. Our account assumes that the rTMS protocol both suppressed activity in the stimulated hemisphere and generated compensatory activation. The effects of inhibition and compensatory activation are then modulated by the corpus callosum.

First, our findings support the link between higher FA/HMOA and greater inhibition of the nondominant hemisphere. Accordingly, in participants with a high FA/HMOA, the right dominant hemisphere directs attention toward the contralateral (left) visual field and strongly inhibits the nondominant left hemisphere. The application of cTBS over the left IPS additionally inhibits the left hemisphere and triggers a leftward attentional shift. The extent of this shift increases with a higher FA/HMOA (Fig. $7 A$, left). In 
A

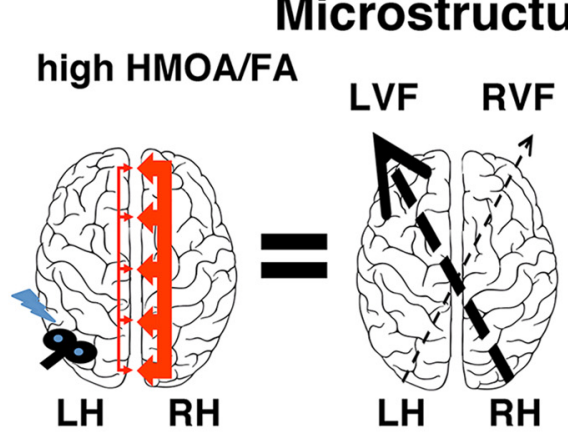

Microstructural organization Iow HMOA/FA

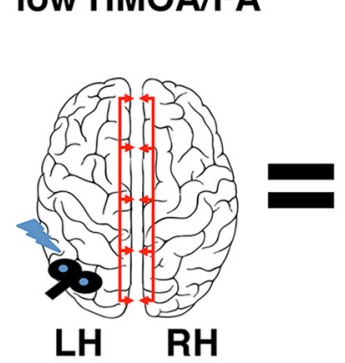

B

\section{Macrostructural organization}

large volume

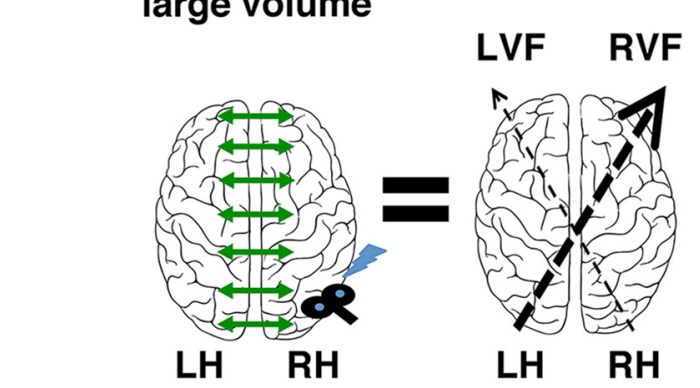

\section{small volume}

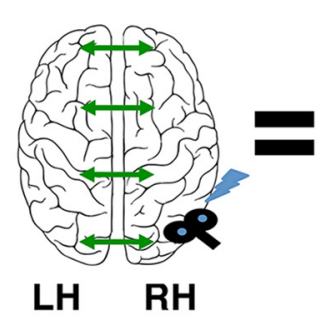

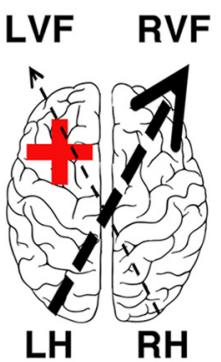

"Typical"
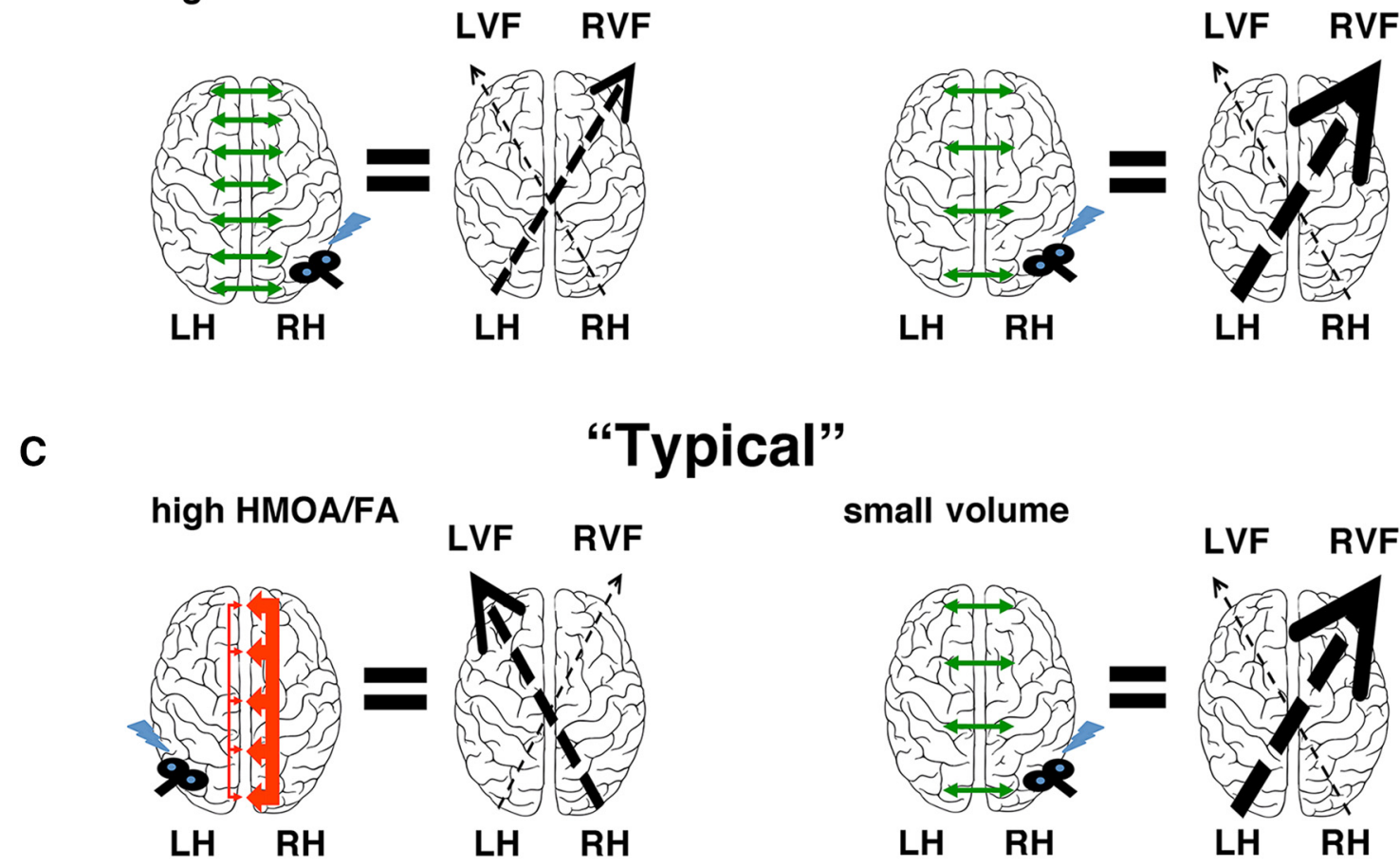

Figure 7. Dual inhibitory and excitatory model of callosal function in spatial attention. $A$, Inhibitory model. cTBS applied over the left IPS in participants with a high FA/HMOA index triggers a leftward attentional shift because the inhibitory stimulation is applied to regions already inhibited by the right dominant hemisphere (left panel). In participants with a low FA/HMOA index, there is a weak reciprocal inhibition of the two hemispheres across the respective attentional networks. CTBS over the left IPS thus triggers compensatory activation of other cortical areas within the left hemisphere (red cross), with the result that spatial attention is directed to the contralateral (right) visual field (right panel). $\boldsymbol{B}$, Excitatory model. In participants with a large corpus callosum (large volume), cTBS applied over the right IPS results in smaller rightward attentional shifts. This is due to the fact that, whereas this stimulation triggers some inhibition of the right hemisphere, there is also high interhemispheric connectivity, causing rebalancing activation across both hemispheres (left panel). In participants with a smaller corpus callosum (small volume), cTBS over the right IPS results in larger rightward attentional shifts due to stimulation triggering inhibition within the dominant right hemisphere and the smaller corpus callosum being less efficient in rebalancing activation across both hemispheres (right panel). C, Dual model. The "typical" responses to cTBS can in turn be better accounted for not by a purely inhibitory model (as originally proposed by Kinsbourne, 1977, 1987, 1993), but rather by a dual inhibitory and excitatory model of callosal function, differentially maintaining right hemispheric dominance in spatial attention.

contrast, in participants with a low FA/HMOA, there is weak reciprocal inhibition of the two hemispheres. When cTBS is applied over the left IPS, we propose that compensatory activation is triggered within the left hemisphere (outside of the IPS) and this directs spatial attention to the contralateral visual field; the result is thus a rightward attentional shift (Fig. $7 A$, right). In contrast, any compensatory ipsilateral activation in participants with a high FA/HMOA would have little effect due to strong inhibition by the dominant right hemisphere. In the current study, we have not examined cortical activity directly, but earlier fMRI studies support our proposal. For instance, higher FA in the corpus callosum has been associated previously with inhibition of cortical activity in the nondominant hemisphere, whereas lower FA in the corpus callosum has been associated with weak reciprocal inhibition and greater activity of the nondominant hemisphere (Putnam et al., 2008). Combined rTMS-fMRI studies have further demonstrated that inhibitory stimulation applied to the left PPC triggers an increase in activity within several frontoparietal regions, including the ipsilateral superior parietal lobule, the bilateral temporoparietal junction, and the bilateral visual cortex (Sack et al., 2007; Ruff et al., 2008, 2009; Plow et al., 2014). These results fit with our proposal that, in participants with weak reciprocal inhibition (a low FA/HMOA), strong compensatory activation is triggered within the left hemisphere and this results 
in a rightward attentional shift. Finally, using a trifocal-TMS stimulation protocol, Koch et al. (2011) have shown a link between FA in the posterior corpus callosum and interhemispheric inhibition. Consistent with our findings, they showed a positive correlation between interhemispheric inhibition of the left PPC by the right PPC and FA (i.e., a higher FA equals higher inhibition).

Second, previous evidence suggests that a smaller corpus callosum is associated with increased functional lateralization (Clarke and Zaidel, 1994; Yazgan et al., 1995). This fits with the excitatory model of callosal function, in which a smaller corpus callosum is associated with reduced interhemispheric connectivity, thus maintaining functional lateralization (Bloom and Hynd, 2005). Here, we propose that the "typical" responses to cTBS, reflecting strong right hemisphere dominance in spatial attention (i.e., rightward attentional shifts after cTBS over the right IPS and leftward attentional shifts after cTBS over the left IPS) are better accounted for not by a purely inhibitory model, as originally proposed by Kinsbourne (1977), but by a dual-model of inhibitory and excitatory functions of the corpus callosum. This model is supported by higher FA/HMOA within the corpus callosum and lower corpus callosum volume in participants with "typical" responses to cTBS (Fig. 7C). Interestingly, we also found a significant negative correlation between the HMOA and the volume within the parietal portion of the corpus callosum interconnecting areas within inferior and superior parietal lobule (i.e., key cortical regions within the dorsal attention network controlling spatial attention).

Our findings that cTBS applied over the right IPS results in smaller rightward attentional shifts in participants with larger corpus callosum are difficult to accommodate in terms of a "purely inhibitory" model. However, the results can be accounted for if high interhemispheric connectivity rebalances activation across both hemispheres after right hemisphere inhibition by cTBS (Fig. 7B, left). In contrast, a smaller corpus callosum should be less efficient in rebalancing activation across both hemispheres after cTBS to the right IPS (Fig. $7 B$, right). We propose that, when cTBS is applied over the right IPS, it triggers both IPS inhibition and compensatory activation within attentional networks outside of the IPS. Indeed, it has been shown that rTMS of the right PPC results in increased activation within several regions, including the bilateral temporoparietal junction (Sack et al., 2007) and bilateral visual cortex (Ruff et al., 2008, 2009). Accordingly, high interhemispheric connectivity (large corpus callosum) facilitates the spreading of both inhibition and compensatory activation across hemispheres and the overall result is thus a smaller rightward attentional shift (Fig. 7B, left). However, in participants with a small corpus callosum, despite compensatory activation, there is no rebalancing after cTBS of the right IPS, so the overall result is a larger rightward attentional shift (Fig. 7B, right). Interestingly, rTMS over the right PPC can trigger, not only compensatory activation, but also decreases in activity in other frontal and parietal regions within the dorsal attention network (i.e., outside the immediate stimulation site; Sack et al., 2007). This result fits well with our proposal that an additional decrease in activity within the dorsal attention network controlling the allocation of spatial attention would be rebalanced in participants with high interhemispheric connectivity and result in smaller rightward attentional shifts. As a caveat, though, please note that our evidence suggesting that the volume of the corpus callosum (macrostructural variability) is a predictor of the magnitude of attentional shifts after cTBS over the right
IPS is weaker than the link between HMOA/FA and response to cTBS over the left IPS.

We suggest that the microstructural and macrostructural organization of the corpus callosum represents inhibitory and excitatory interactions between the cerebral hemispheres. The link between the macrostructural organization of the corpus callosum and interhemispheric transfer is consistent with the concept that a smaller corpus callosum increases functional lateralization by decreasing interhemispheric connectivity. Anatomical studies indicate that the size of the corpus callosum depends on the number of thin (small diameter) fibers, which comprise the majority of the callosal body and interconnect homologous cortical regions (Aboitiz et al., 1992a,b; Aboitiz and Montiel, 2003). The relationship between track volume (calculated here as the number of voxels intersected by the reconstructed streamlines) and actual axonal number, axonal diameter, and density has yet to be established (Beaulieu, 2002). The remaining question is how to reconcile these findings with high FA/HMOA supporting greater inhibition of nondominant hemisphere. Although the inhibitory model assumes that greater inhibition and lateralization depends on stronger connectivity, there is no evidence to support a linear relationship between FA/HMOA and connectivity (i.e., that higher FA/HMOA is equivalent to higher connectivity; Beaulieu, 2002; Dell'acqua et al., 2013; Jones et al., 2013). Therefore, we suggest that the link between the FA/HMOA and inhibition of nondominant hemisphere does not rely on connectivity per se and that high FA/HMOA represents a surrogate of tissue microstructure influencing functional hemispheric inhibition. Finally, it should be noted that the volume and FA/HMOA clearly account for different properties of the WM and likely reflect contrasting aspects of the corpus callosum function.

In conclusion, our data indicate that microstructural and macrostructural individual variability within the corpus callosum determine the response to cTBS over the IPS and these two factors differentially mediate interhemispheric dynamics. Our findings suggest that interhemispheric dynamics and communication via the corpus callosum in directing attention in space are not purely based on inhibition, but may be better explained by a dual model in which the structural organization of the corpus callosum dynamically reinforces both interhemispheric inhibition and information transfer between hemispheres underlying hemispheric lateralization in visuospatial attention. We propose the following: (1) that the contribution of the microstructural and macrostructural organization of the corpus callosum to hemispheric lateralization in spatial attention differs significantly on an individual level and (2) that these differences putatively reflect inhibitory and excitatory mechanisms of callosal function that are not completely independent, but work together in maintaining functional lateralization. Finally, our data provide some evidence of regional specificity within the corpus callosum and for the dual model of callosal function while also suggesting that, outside of the parietal cortex, other cortical areas interact competitively (Hilgetag et al., 2001) via the corpus callosum.

\section{References}

Aboitiz F, Montiel J (2003) One hundred million years of interhemispheric communication: the history of the corpus callosum. Braz J Med Biol Res 36:409-420. Medline

Aboitiz F, Scheibel AB, Fisher RS, Zaidel E (1992a) Fiber composition of the human corpus callosum. Brain Res 598:143-153. CrossRef Medline

Aboitiz F, Scheibel AB, Fisher RS, Zaidel E (1992b) Individual differences in brain asymmetries and fiber composition in the human corpus callosum. Brain Res 598:154-161. CrossRef Medline

Allen LS, Richey MF, Chai YM, Gorski RA (1991) Sex differences in the 
corpus callosum of the living human being. J Neurosci 11:933-942. Medline

Andersson JLR, Sotiropoulos SN (2015a) An integrated approach to correction for off-resonance effects and subject movement in diffusion MR imaging. Neuroimage. In press.

Andersson JL, Sotiropoulos SN (2015b) Non-parametric representation and prediction of single- and multi-shell diffusion-wighted MRI using Gaussian processes. Neuroimage 122:166-176. CrossRef Medline

Andersson JL, Skare S, Ashburner J (2003) How to correct susceptibility distortions in spin-echo echo-planar images: application to diffusion tensor imaging. Neuroimage 20:870-888. CrossRef Medline

Anstey KJ, Mack HA, Christensen H, Li SC, Reglade-Meslin C, Maller J, Kumar R, Dear K, Easteal S, Sachdev P (2007) Corpus callosum size, reaction time speed and variability in mild cognitive disorders and in a normative sample. Neuropsychologia 45:1911-1920. CrossRef Medline

Ardekani BA, Figarsky K, Sidtis JJ (2013) Sexual dimorphism in the human corpus callosum: an MRI study using the OASIS brain database. Cereb Cortex 23:2514-2520. CrossRef Medline

Basser PJ, Mattiello J, LeBihan D (1994) MR diffusion tensor spectroscopy and imaging. Biophys J 66:259-267. CrossRef Medline

Battelli L, Alvarez GA, Carlson T, Pascual-Leone A (2009) The role of the parietal lobe in visual extinction studied with transcranial magnetic stimulation. J Cogn Neurosci 21:1946-1955. CrossRef Medline

Beaulieu C (2002) The basis of anisotropic water diffusion in the nervous system-a technical review. NMR Biomed 15:435-455. CrossRef Medline

Benjamini Y, Hochberg Y (1995) Controlling the false discovery rate: a practical and powerful approach to multiple testing. Journal of the Royal Statistical Society Series B (Methodological) 57:289-300.

Bishop KM, Wahlsten D (1997) Sex differences in the human corpus callosum: myth or reality? Neurosci Biobehav Rev 21:581-601. CrossRef Medline

Bjoertomt O, Cowey A, Walsh V (2002) Spatial neglect in near and far space investigated by repetitive transcranial magnetic stimulation. Brain 125: 2012-2022. CrossRef Medline

Bloom JS, Hynd GW (2005) The role of the corpus callosum in interhemispheric transfer of information: excitation or inhibition? Neuropsychol Rev 15:59-71. CrossRef Medline

Bowers D, Heilman KM (1980) Pseudoneglect: effects of hemispace on a tactile line bisection task. Neuropsychologia 18:491-498. CrossRef Medline

Bryden MP, Hécaen H, DeAgostini M (1983) Patterns of cerebral organization. Brain Lang 20:249-262. CrossRef Medline

Cai Q, Van der Haegen L, Brysbaert M (2013) Complementary hemispheric specialization for language production and visuospatial attention. Proc Natl Acad Sci U S A 110:E322-E330. CrossRef Medline

Carter AR, Astafiev SV, Lang CE, Connor LT, Rengachary J, Strube MJ, Pope DL, Shulman GL, Corbetta M (2010) Resting interhemispheric functional magnetic resonance imaging connectivity predicts performance after stroke. Ann Neurol 67:365-375. Medline

Cazzoli D, Wurtz P, Müri RM, Hess CW, Nyffeler T (2009) Interhemispheric balance of overt attention: a theta burst stimulation study. Eur J Neurosci 29:1271-1276. CrossRef Medline

Cazzoli D, Müri RM, Hess CW, Nyffeler T (2010) Treatment of hemispatial neglect by means of rTMS-a review. Restor Neurol Neurosci 28:499-510. Medline

Cazzoli D, Müri RM, Schumacher R, von Arx S, Chaves S, Gutbrod K, Bohlhalter S, Bauer D, Vanbellingen T, Bertschi M, Kipfer S, Rosenthal CR, Kennard C, Bassetti CL, Nyffeler T (2012) Theta burst stimulation reduces disability during the activities of daily living in spatial neglect. Brain 135:3426-3439. CrossRef Medline

Chang H, Fitzpatrick JM (1992) A technique for accurate magnetic resonance imaging in the presence of field inhomogeneities. IEEE Trans Med Imaging 11:319-329. CrossRef Medline

Chechlacz M, Gillebert CR, Vangkilde SA, Petersen A, Humphreys GW (2015) Structural variability within frontoparietal networks and individual differences in attentional functions captured by Bundesen's theory of visual attention. J Neurosci 35:10647-10658. CrossRef Medline

Clarke JM, Zaidel E (1994) Anatomical-behavioral relationships: corpus callosum morphometry and hemispheric specialization. Behav Brain Res 64:185-202. CrossRef Medline

Collins DL, Holmes CJ, Peters TM, Evans AC (1995) Automatic 3-D model- based neuroanatomical segmentation. Hum Brain Mapp 3:190-208. CrossRef

Corbetta M, Shulman GL (2002) Control of goal-directed and stimulusdriven attention in the brain. Nat Rev Neurosci 3:201-215. Medline

Corbetta M, Shulman GL (2011) Spatial neglect and attention networks. Annu Rev Neurosci 34:569-599. CrossRef Medline

Corbetta M, Kincade MJ, Lewis C, Snyder AZ, Sapir A (2005) Neural basis and recovery of spatial attention deficits in spatial neglect. Nat Neurosci 8:1603-1610. CrossRef Medline

Corbetta M, Ramsey L, Callejas A, Baldassarre A, Hacker CD, Siegel JS, Astafiev SV, Rengachary J, Zinn K, Lang CE, Connor LT, Fucetola R, Strube M, Carter AR, Shulman GL (2015) Common behavioral clusters and subcortical anatomy in stroke. Neuron 85:927-941. CrossRef Medline

Crosby EC (1962) Correlative anatomy of the nervous system. New York: Macmillan.

Dambeck N, Sparing R, Meister IG, Wienemann M, Weidemann J, Topper R, Boroojerdi B (2006) Interhemispheric imbalance during visuospatial attention investigated by unilateral and bilateral TMS over human parietal cortices. Brain Res 1072:194-199. CrossRef Medline

Dell'acqua F, Scifo P, Rizzo G, Catani M, Simmons A, Scotti G, Fazio F (2010) A modified damped Richardson-Lucy algorithm to reduce isotropic background effects in spherical deconvolution. Neuroimage 49: 1446-1458. CrossRef Medline

Dell'acqua F, Simmons A, Williams SC, Catani M (2013) Can spherical deconvolution provide more information than fiber orientations? Hindrance modulated orientational anisotropy, a true-tract specific index to characterize white matter diffusion. Hum Brain Mapp 34:2464-2483. CrossRef Medline

Denenberg VH, Kertesz A, Cowell PE (1991) A factor analysis of the human's corpus callosum. Brain Res 548:126-132. CrossRef Medline

Desikan RS, Ségonne F, Fischl B, Quinn BT, Dickerson BC, Blacker D, Buckner RL, Dale AM, Maguire RP, Hyman BT, Albert MS, Killiany RJ (2006) An automated labeling system for subdividing the human cerebral cortex on MRI scans into gyral based regions of interest. Neuroimage 31:968980. CrossRef Medline

Driver J, Mattingley JB (1998) Parietal neglect and visual awareness. Nat Neurosci 1:17-22. CrossRef Medline

Dubb A, Gur R, Avants B, Gee J (2003) Characterization of sexual dimorphism in the human corpus callosum. Neuroimage 20:512-519. CrossRef Medline

Duecker F, Sack AT (2015) The hybrid model of attentional control: New insights into hemispheric asymmetries inferred from TMS research. Neuropsychologia 74:21-29. CrossRef Medline

Duecker F, Formisano E, Sack AT (2013) Hemispheric differences in the voluntary control of spatial attention: direct evidence for a righthemispheric dominance within frontal cortex. J Cogn Neurosci 25: 1332-1342. CrossRef Medline

Fierro B, Brighina F, Oliveri M, Piazza A, La Bua V, Buffa D, Bisiach E (2000) Contralateral neglect induced by right posterior parietal rTMS in healthy subjects. Neuroreport 11:1519-1521. CrossRef Medline

Flöel A, Buyx A, Breitenstein C, Lohmann H, Knecht S (2005a) Hemispheric lateralization of spatial attention in right- and left-hemispheric language dominance. Behav Brain Res 158:269-275. CrossRef Medline

Flöel A, Jansen A, Deppe M, Kanowski M, Konrad C, Sommer J, Knecht S (2005b) Atypical hemispheric dominance for attention: functional MRI topography. J Cereb Blood Flow Metab 25:1197-1208. CrossRef Medline

Gillebert CR, Mantini D, Thijs V, Sunaert S, Dupont P, Vandenberghe R (2011) Lesion evidence for the critical role of the intraparietal sulcus in spatial attention. Brain 134:1694-1709. CrossRef Medline

Glasser MF, Sotiropoulos SN, Wilson JA, Coalson TS, Fischl B, Andersson JL, Xu J, Jbabdi S, Webster M, Polimeni JR, Van Essen DC, Jenkinson M; WU-Minn HCP Consortium (2013) The minimal preprocessing pipelines for the Human Connectome Project. Neuroimage 80:105-124. CrossRef Medline

Goldsworthy MR, Pitcher JB, Ridding MC (2012) A comparison of two different continuous theta burst stimulation paradigms applied to the human primary motor cortex. Clin Neurophysiol 123:2256-2263. CrossRef Medline

Habib M, Gayraud D, Oliva A, Regis J, Salamon G, Khalil R (1991) Effects of handedness and sex on the morphology of the corpus callosum: a study with brain magnetic resonance imaging. Brain Cogn 16:41-61. CrossRef Medline 
Halligan PW, Fink GR, Marshall JC, Vallar G (2003) Spatial cognition: evidence from visual neglect. Trends Cogn Sci 7:125-133. CrossRef Medline

Heilman KM, Valenstein E (1979) Mechanisms underlying hemispatial neglect. Ann Neurol 5:166-170. CrossRef Medline

Hilgetag CC, Théoret H, Pascual-Leone A (2001) Enhanced visual spatial attention ipsilateral to rTMS-induced 'virtual lesions' of human parietal cortex. Nat Neurosci 4:953-957. CrossRef Medline

Hofer S, Frahm J (2006) Topography of the human corpus callosum revisited-comprehensive fiber tractography using diffusion tensor magnetic resonance imaging. Neuroimage 32:989-994. CrossRef Medline

Hua K, Zhang J, Wakana S, Jiang H, Li X, Reich DS, Calabresi PA, Pekar JJ, van Zijl PC, Mori S (2008) Tract probability maps in stereotaxic spaces: Analyses of white matter anatomy and tract-specific quantification. Neuroimage 39:336-347. CrossRef Medline

Huang YZ, Edwards MJ, Rounis E, Bhatia KP, Rothwell JC (2005) Theta burst stimulation of the human motor cortex. Neuron 45:201-206. CrossRef Medline

Hung J, Driver J, Walsh V (2005) Visual selection and posterior parietal cortex: effects of repetitive transcranial magnetic stimulation on partial report analyzed by Bundesen's theory of visual attention. J Neurosci 25: 9602-9612. CrossRef Medline

Jäncke L, Staiger JF, Schlaug G, Huang Y, Steinmetz H (1997) The relationship between corpus callosum size and forebrain volume. Cereb Cortex 7:48-56. CrossRef Medline

Jones DK, Knösche TR, Turner R (2013) White matter integrity, fiber count, and other fallacies: the do's and don'ts of diffusion MRI. Neuroimage 73:239-254. CrossRef Medline

Josse G, Seghier ML, Kherif F, Price CJ (2008) Explaining function with anatomy: language lateralization and corpus callosum size. J Neurosci 28:14132-14139. CrossRef Medline

Kertesz A, Polk M, Howell J, Black SE (1987) Cerebral dominance, sex, and callosal size in MRI. Neurology 37:1385-1388. CrossRef Medline

Kinsbourne, M (1977) Hemi-neglect and hemisphere rivalry. In: Hemiinattention and hemisphere specialization (Weinstein EA, Friedland RP, eds), pp 41-49. New York: Raven.

Kinsbourne, M (1987) Mechanisms of unilateral neglect. In: Neurophysiological and neuropsychological aspects of spatial neglect (Jeannerod M, ed), pp 69-86. Amsterdam: Elsevier Science.

Kinsbourne, M (1993) Orientational bias model of unilateral neglect: Evidence from attentional gradients within hemispace. In: Unilateral neglect: clinical and experimental studies (Robertson IH, Marshall JC, eds), pp 63-86. Hove, UK: Lawrence Erlbaum Associates.

Koch G, Cercignani M, Bonnì S, Giacobbe V, Bucchi G, Versace V, Caltagirone C, Bozzali M (2011) Asymmetry of parietal interhemispheric connections in humans. J Neurosci 31:8967-8975. CrossRef Medline

Koch G, Bonnì S, Giacobbe V, Bucchi G, Basile B, Lupo F, Versace V, Bozzali M, Caltagirone C (2012) theta-burst stimulation of the left hemisphere accelerates recovery of hemispatial neglect. Neurology 78:24-30. CrossRef Medline

Landis JR, Koch GG (1977a) The measurement of observer agreement for categorical data. Biometrics 33:159-174. CrossRef Medline

Landis JR, Koch GG (1977b) An application of hierarchical kappa-type statistics in the assessment of majority agreement among multiple observers. Biometrics 33:363-374. CrossRef Medline

Luders E, Rex DE, Narr KL, Woods RP, Jäncke L, Thompson PM, Mazziotta JC, Toga AW (2003) Relationships between sulcal asymmetries and corpus callosum size: gender and handedness effects. Cereb Cortex 13: 1084-1093. CrossRef Medline

Luders E, Cherbuin N, Thompson PM, Gutman B, Anstey KJ, Sachdev P, Toga AW (2010) When more is less: associations between corpus callosum size and handedness lateralization. Neuroimage 52:43-49. CrossRef Medline

Lunven M, Thiebaut De Schotten M, Bourlon C, Duret C, Migliaccio R, Rode G, Bartolomeo P (2015) White matter lesional predictors of chronic visual neglect: a longitudinal study. Brain 138:746-760. CrossRef Medline

Marshall TR, O'Shea J, Jensen O, Bergmann TO (2015) Frontal eye fields control attentional modulation of alpha and gamma oscillations in contralateral occipitoparietal cortex. J Neurosci 35:1638-1647. CrossRef Medline

Mazoyer B, Zago L, Jobard G, Crivello F, Joliot M, Perchey G, Mellet E, Petit L, Tzourio-Mazoyer N (2014) Gaussian mixture modeling of hemispheric lateralization for language in a large sample of healthy individuals balanced for handedness. PLoS One 9:e101165. CrossRef Medline
Mazziotta J, Toga A, Evans A, Fox P, Lancaster J, Zilles K, Woods R, Paus T, Simpson G, Pike B, Holmes C, Collins L, Thompson P, MacDonald D, Iacoboni M, Schormann T, Amunts K, Palomero-Gallagher N, Geyer S, Parsons L, et al. (2001) A probabilistic atlas and reference system for the human brain: International Consortium for Brain Mapping (ICBM). Philos Trans R Soc Lond B Biol Sci 356:1293-1322. CrossRef Medline

McCourt ME, Jewell G (1999) Visuospatial attention in line bisection: stimulus modulation of pseudoneglect. Neuropsychologia 37:843-855. CrossRef Medline

Mesulam MM (1990) Large-scale neurocognitive networks and distributed processing for attention, language, and memory. Ann Neurol 28: 597-613. CrossRef Medline

Mori S (2005) MRI atlas of human white matter. Amsterdam: Elsevier.

Nichols TE, Holmes AP (2002) Nonparametric permutation tests for functional neuroimaging: a primer with examples. Hum Brain Mapp 15:1-25. CrossRef Medline

Nicolo P, Ptak R, Guggisberg AG (2015) Variability of behavioural responses to transcranial magnetic stimulation: Origins and predictors. Neuropsychologia.

Nyffeler T, Cazzoli D, Wurtz P, Lüthi M, von Wartburg R, Chaves S, Déruaz A, Hess CW, Müri RM (2008) Neglect-like visual exploration behaviour after theta burst transcranial magnetic stimulation of the right posterior parietal cortex. Eur J Neurosci 27:1809-1813. CrossRef Medline

Nyffeler T, Cazzoli D, Hess CW, Müri RM (2009) One session of repeated parietal theta burst stimulation trains induces long-lasting improvement of visual neglect. Stroke 40:2791-2796. CrossRef Medline

Oldfield RC (1971) The assessment and analysis of handedness: the Edinburgh inventory. Neuropsychologia 9:97-113. CrossRef Medline

Park HJ, Kim JJ, Lee SK, Seok JH, Chun J, Kim DI, Lee JD (2008) Corpus callosal connection mapping using cortical gray matter parcellation and DT-MRI. Hum Brain Mapp 29:503-516. CrossRef Medline

Petit L, Zago L, Mellet E, Jobard G, Crivello F, Joliot M, Mazoyer B, TzourioMazoyer N (2015) Strong rightward lateralization of the dorsal attentional network in left-handers with right sighting-eye: an evolutionary advantage. Hum Brain Mapp 36:1151-1164. CrossRef Medline

Plow EB, Cattaneo Z, Carlson TA, Alvarez GA, Pascual-Leone A, Battelli L (2014) The compensatory dynamic of inter-hemispheric interactions in visuospatial attention revealed using rTMS and fMRI. Front Hum Neurosci 8:226. Medline

Pollmann S (2004) Anterior prefrontal cortex contributions to attention control. Exp Psychol 51:270-278. CrossRef Medline

Pollmann S (2012) Anterior prefrontal contributions to implicit attention control. Brain Sci 2:254-266. CrossRef Medline

Preuss UW, Meisenzahl EM, Frodl T, Zetzsche T, Holder J, Leinsinger G, Hegerl U, Hahn K, Möller HJ (2002) Handedness and corpus callosum morphology. Psychiatry Res 116:33-42. CrossRef Medline

Putnam MC, Wig GS, Grafton ST, Kelley WM, Gazzaniga MS (2008) Structural organization of the corpus callosum predicts the extent and impact of cortical activity in the nondominant hemisphere. J Neurosci 28:29122918. CrossRef Medline

Quentin R, Chanes L, Vernet M, Valero-Cabré A (2015) Fronto-parietal anatomical connections influence the modulation of conscious visual perception by high-beta frontal oscillatory activity. Cereb Cortex 25: 2095-2101. CrossRef Medline

Rizk S, Ptak R, Nyffeler T, Schnider A, Guggisberg AG (2013) Network mechanisms of responsiveness to continuous theta-burst stimulation. Eur J Neurosci 38:3230-3238. CrossRef Medline

Rojkova K, Volle E, Urbanski M, Humbert F, Dell'acqua F, Thiebaut de Schotten M (2015) Atlasing the frontal lobe connections and their variability due to age and education: a spherical deconvolution tractography study. Brain Struct Funct. In press.

Rossi S, Hallett M, Rossini PM, Pascual-Leone A; Safety of TMS Consensus Group (2009) Safety, ethical considerations, and application guidelines for the use of transcranial magnetic stimulation in clinical practice and research. Clin Neurophysiol 120:2008-2039. CrossRef Medline

Ruff CC, Bestmann S, Blankenburg F, Bjoertomt O, Josephs O, Weiskopf N, Deichmann R, Driver J (2008) Distinct causal influences of parietal versus frontal areas on human visual cortex: evidence from concurrent TMSfMRI. Cereb Cortex 18:817-827. CrossRef Medline

Ruff CC, Blankenburg F, Bjoertomt O, Bestmann S, Weiskopf N, Driver J (2009) Hemispheric differences in frontal and parietal influences on 
human occipital cortex: direct confirmation with concurrent TMS-fMRI. J Cogn Neurosci 21:1146-1161. CrossRef Medline

Sack AT, Kohler A, Bestmann S, Linden DE, Dechent P, Goebel R, Baudewig J (2007) Imaging the brain activity changes underlying impaired visuospatial judgments: simultaneous FMRI, TMS, and behavioral studies. Cereb Cortex 17:2841-2852. CrossRef Medline

Salvador R, Silva S, Basser PJ, Miranda PC (2011) Determining which mechanisms lead to activation in the motor cortex: a modeling study of transcranial magnetic stimulation using realistic stimulus waveforms and sulcal geometry. Clin Neurophysiol 122:748-758. CrossRef Medline

Shapiro S, Wilk M (1965) An analysis of variance test for normality (complete samples). Biometrika 52:591-611.

Shulman GL, Pope DL, Astafiev SV, McAvoy MP, Snyder AZ, Corbetta M (2010) Right hemisphere dominance during spatial selective attention and target detection occurs outside the dorsal frontoparietal network. J Neurosci 30:3640-3651. CrossRef Medline

Silver MA, Kastner S (2009) Topographic maps in human frontal and parietal cortex. Trends Cogn Sci 13:488-495. CrossRef Medline

Smith SM, Nichols TE (2009) Threshold-free cluster enhancement: addressing problems of smoothing, threshold dependence and localisation in cluster inference. Neuroimage 44:83-98. CrossRef Medline

Smith SM, Jenkinson M, Woolrich MW, Beckmann CF, Behrens TE, Johansen-Berg H, Bannister PR, De Luca M, Drobnjak I, Flitney DE, Niazy RK, Saunders J, Vickers J, Zhang Y, De Stefano N, Brady JM, Matthews PM (2004) Advances in functional and structural MR image analysis and implementation as FSL. Neuroimage 23:S208-S219. CrossRef Medline

Smith SM, Jenkinson M, Johansen-Berg H, Rueckert D, Nichols TE, Mackay CE, Watkins KE, Ciccarelli O, Cader MZ, Matthews PM, Behrens TE (2006) Tract-based spatial statistics: voxelwise analysis of multi-subject diffusion data. Neuroimage 31:1487-1505. CrossRef Medline

Somers M, Shields LS, Boks MP, Kahn RS, Sommer IE (2015) Cognitive benefits of right-handedness: a meta-analysis. Neurosci Biobehav Rev 51:48-63. CrossRef Medline

Sosa Y, Teder-Sälejärvi WA, McCourt ME (2010) Biases of spatial attention in vision and audition. Brain Cogn 73:229-235. CrossRef Medline

Sotiropoulos SN, Jbabdi S, Xu J, Andersson JL, Moeller S, Auerbach EJ, Glasser MF, Hernandez M, Sapiro G, Jenkinson M, Feinberg DA, Yacoub E, Lenglet C, Van Essen DC, Ugurbil K, Behrens TE; WU-Minn HCP Consortium (2013) Advances in diffusion MRI acquisition and processing in the Human Connectome Project. Neuroimage 80:125-143. CrossRef Medline

Szaflarski JP, Binder JR, Possing ET, McKiernan KA, Ward BD, Hammeke TA (2002) Language lateralization in left-handed and ambidextrous people: fMRI data. Neurology 59:238-244. CrossRef Medline

Szczepanski SM, Kastner S (2009) Transcranial magnetic stimulation studies of visuospatial attentional control. F1000 Biol Rep 1:81. Medline

Szczepanski SM, Kastner S (2013) Shifting attentional priorities: control of spatial attention through hemispheric competition. J Neurosci 33: 5411-5421. CrossRef Medline

Szczepanski SM, Konen CS, Kastner S (2010) Mechanisms of spatial attention control in frontal and parietal cortex. J Neurosci 30:148-160. CrossRef Medline

Szczepanski SM, Pinsk MA, Douglas MM, Kastner S, Saalmann YB (2013) Functional and structural architecture of the human dorsal frontoparietal attention network. Proc Natl Acad Sci U S A 110:15806-15811. CrossRef Medline

Thiebaut de Schotten M, Dell'acqua F, Forkel SJ, Simmons A, Vergani F, Murphy DG, Catani M (2011a) A lateralized brain network for visuospatial attention. Nat Neurosci 14:1245-1246. CrossRef Medline

Thiebaut de Schotten M, Ffytche DH, Bizzi A, Dell'acqua F, Allin M, Walshe M, Murray R, Williams SC, Murphy DG, Catani M (2011b) Atlasing location, asymmetry and inter-subject variability of white matter tracts in the human brain with MR diffusion tractography. Neuroimage 54:49-59. CrossRef Medline

Thiebaut de Schotten M, Dell'acqua F, Valabregue R, Catani M (2012) Monkey to human comparative anatomy of the frontal lobe association tracts. Cortex 48:82-96. CrossRef Medline

Tuncer MC, Hatipoğlu ES, Ozateş M (2005) Sexual dimorphism and handedness in the human corpus callosum based on magnetic resonance imaging. Surg Radiol Anat 27:254-259. CrossRef Medline

Vallar G (1998) Spatial hemineglect in humans. Trends Cogn Sci 2:87-97. CrossRef Medline

van der Knaap LJ, van der Ham IJ (2011) How does the corpus callosum mediate interhemispheric transfer? A review. Behav Brain Res 223: 211-221. CrossRef Medline

Whitehouse AJ, Badcock N, Groen MA, Bishop DV (2009) Reliability of a novel paradigm for determining hemispheric lateralization of visuospatial function. J Int Neuropsychol Soc 15:1028-1032. CrossRef Medline

Willems RM, Van der Haegen L, Fisher SE, Francks C (2014) On the other hand: including left-handers in cognitive neuroscience and neurogenetics. Nat Rev Neurosci 15:193-201. Medline

Winkler AM, Ridgway GR, Webster MA, Smith SM, Nichols TE (2014) Permutation inference for the general linear model. Neuroimage 92:381-397. CrossRef Medline

Witelson SF (1985) The brain connection: the corpus callosum is larger in left-handers. Science 229:665-668. CrossRef Medline

Witelson SF (1989) Hand and sex differences in the isthmus and genu of the human corpus callosum: a postmortem morphological study. Brain 112: 799-835. CrossRef Medline

Yazgan MY, Wexler BE, Kinsbourne M, Peterson B, Leckman JF (1995) Functional significance of individual variations in callosal area. Neuropsychologia 33:769-779. CrossRef Medline

Zarei M, Johansen-Berg H, Smith S, Ciccarelli O, Thompson AJ, Matthews PM (2006) Functional anatomy of interhemispheric cortical connections in the human brain. J Anat 209:311-320. CrossRef Medline 